\title{
Review
}

\section{Cosmoparticle Physics of Dark Universe}

\author{
Maxim Khlopov 1,2,3
}

check for updates

Citation: Khlopov, M. Cosmoparticle Physics of Dark Universe. Symmetry 2022, 14, 112. https://doi.org/ 10.3390/sym14010112

Academic Editor: Alexander B. Balakin

Received: 30 November 2021

Accepted: 5 January 2022

Published: 9 January 2022

Publisher's Note: MDPI stays neutral with regard to jurisdictional claims in published maps and institutional affiliations.

Copyright: (c) 2022 by the author. Licensee MDPI, Basel, Switzerland. This article is an open access article distributed under the terms and conditions of the Creative Commons Attribution (CC BY) license (https:// creativecommons.org/licenses/by/ $4.0 /)$.
1 Virtual Institute of Astroparticle Physics, Université de Paris, CNRS, Astroparticule et Cosmologie, F-75013 Paris, France; khlopov@apc.univ-paris7.fr; Tel.: +33-676-380-567

2 Center for Cosmoparticle Physics "Cosmion", National Research Nuclear University, 115409 Moscow, Russia

3 Research Institute of Physics, Sousthern Federal University, Stachki 194, 344090 Rostov on Don, Russia

\begin{abstract}
The physics of the dark Universe goes beyond the standard model (BSM) of fundamental interactions. The now-standard cosmology involves inflation, baryosynthesis and dark matter/energy corresponding to BSM physics. Cosmoparticle physics offers cross disciplinary study of the fundamental relationship of cosmology and particle physics in the combination of its physical, astrophysical and cosmological signatures. Methods of cosmoparticle physics in studies of BSM physics in its relationship with inevitably nonstandard features of dark universe cosmology are discussed. In the context of these methods, such exotic phenomena as primordial black holes, antimatter stars in baryon asymmetrical Universe or multi-charged constituents of nuclear interacting atoms of composite dark matter play the role of sensitive probes for BSM models and their parameters.
\end{abstract}

Keywords: elementary particles; universe; cosmology; inflation; baryosynthesis; dark matter; early universe; symmetry breaking; phase transitions; primordial black holes; antimatter; dark atoms

\section{Introduction}

The two observational features of the Universe-its expansion and the presence of thermal electromagnetic background radiation-were combined in physical picture of G.Gamov's Big Bang Universe. This picture of thermal cosmological history resulted from successive application of known physical laws of thermodynamics, atomic and nuclear physics to the expanding space-time of Friedman-Lemaitre-Robertson-Walker geometry described by non-stationary solutions of General Relativity for homogeneous and isotropic world. Only four known fundamental interactions and the set of known elementary particles were involved in the corresponding cosmological scenario, tracing the creation of the observed Universe in the result of thermal evolution from the initial state of hot plasma with sub-Planckean temperature [1,2]. However, the development cosmological scenarios based on the predictions of particle theory combined with the data of precision cosmology lead to the modern paradigm of inflationary cosmology with baryosynthesis and dark matter/energy dominating in the modern Universe (see, References [3-24] for review and reference).

The data of precision cosmology favor now-standard inflationary cosmology with baryosynthesis and dark matter/energy [3,4,20-23,25-29]. This standard cosmological paradigm involves physics beyond the Standard model (BSM) with new physical scale $V$, which is accessible for combined probes by cosmological, astrophysical and experimental physical means as illustrated on Figure 1. 


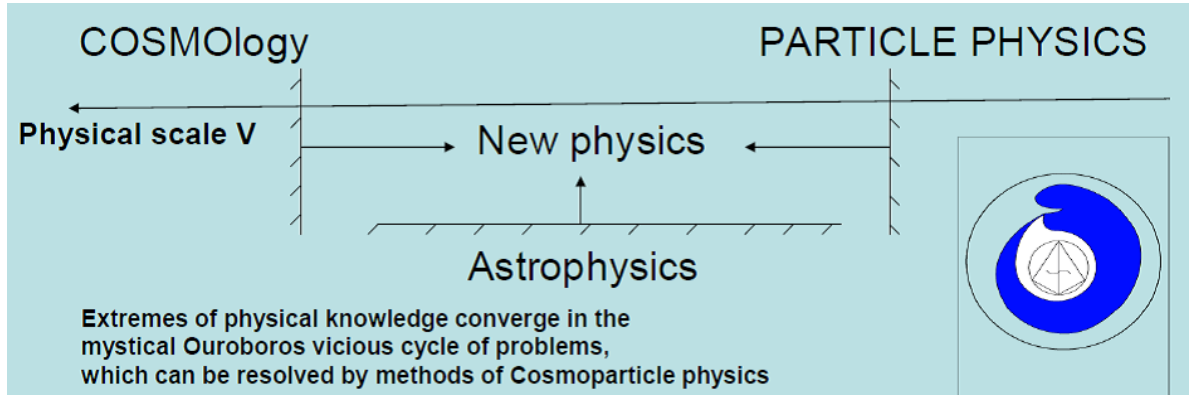

Figure 1. Cosmoparticle physics probes the scale $V$ of new physics by proper combination of its cosmological astrophysical and experimental physical signatures. The author's original picture from [29].

In the context of BSM models, dark matter candidates are related to predictions of new stable or long living particles or macroscopic structures, which reflect the fundamental structure of this model [8-12,25,29-32]. Stability of new particles reflects new strict (or approximate) BSM symmetry that leads to new conserved charges, making stable (metastable) the lightest particle, which possess this charge. Such a particle can be considered as dark matter (DM) candidate. This prediction is accompanied by a set of other specific modeldependent signatures so that their combination can provide verification of the proposed physical nature of dark matter.

We discuss in the present review the problem of the physical nature of dark Universe, paying special attention to the fundamental physical motivations for SM extensions. We show that DM candidates, predicted by such extensions involve the set of model-dependent signatures of the corresponding BSM model, which accompany the prediction of the DM candidate. We call these accompanying model-dependent phenomena messengers of the dark Universe. In particular such signatures can include set of additional particles and/or new interactions predicted along with the considered dark matter candidate.

In addition to new particles and fields BSM physics involves mechanisms of symmetry breaking that can lead to phase transitions in the early Universe, in which various forms of topological defects can be formed. The high-energy sector of a BSM model can lead to nontrivial scenarios of very early Universe at inflational or post-inflational stage, at which primordial black holes (PBH), $\mathrm{PBH}$ clusters or other forms of primordial nonlinear structures like antimatter domains in baryon asymmetrical Universe can appear.

Here, we concentrate on the case of extensions of symmetry and particle content of the standard model (SM) of elementary particles. Particle dark matter candidates should be absolutely stable or sufficiently long living to provide their existence and dynamical role in cosmological large-scale structure formation. They should be massive and created in the early Universe in the amount, corresponding to the observed dark matter density.

Therefore the BSM model predicting such candidates and mechanisms of their production in the early Universe should involve mechanism of stability of DM particles, preventing their rapid decay. From particle physics viewpoint it means that these particles possess some new conserved quantum number originated from the BSM symmetry. Such new symmetry may be simply added by hands to the SM symmetry together with set of new particles, on which it acts. However, the additional symmetry may be motivated by practical necessity to solve the problems of SM internal inconsistency or incompleteness and such examples are aesthetically much more attractive.

BSM physics of dark Universe can be also originated beyond the standard model of space-time and gravity and related to the effects of extra dimensions or follow from the modified gravity. These topics are beyond the scope of the present review, but the idea of multimessenger probes and methods of cosmoparticle physics are also appropriate for studies of these extensions.

The basic elements of modern cosmology, describing the initial conditions and the modern stage of cosmological evolution-inflation, baryosynthesis and dark matter/energy- 
are related to physics beyond the standard model of fundamental interactions, making the Universe dark from the beginning of Big Bang Universe to the present time. This physics, predicted by extensions of the Standard model of electroweak and strong interactions and/or modified gravity, is beyond the scope of direct experimental means and implies the combination of indirect physical, astrophysical and cosmological probes, developed by cosmoparticle physics [3,4].

Any extension of the Standard Model, which describes these necessary basic elements of modern cosmology, such extension inevitably contains some additional modeldependent messengers of the corresponding physical model here we would like to discuss [25] various forms of such additional cosmological reflections of the fundamental particle symmetry. The presented list of nontrivial examples of such reflections-being far from complete-is challenging for the development of astrophysical, astroparticle, cosmological, and collider probes for new physics.

In Section 2, we present examples of particle dark matter candidates, strongly motivated by the BSM solutions of the internal problems of the Standard model. We consider possibility of their test in the combination of physical, astrophysical and cosmological messengers of the corresponding models. The lack of evidence for supersymmetric solution for the problem of divergence of the Higgs boson mass may favor nonsupersymmetric solution of this problem in models of composite Higgs boson with multiple charged constituents (Section 3). Such may predict heavy stable $-2 n$ charged lepton-like particles, which can be bound with $n$ nuclei of primordial helium in nuclear-interacting dark atoms. The qualitative advantages of using this dark atom scenario to explain several puzzles of direct and indirect searches for dark matter challenge search for stable multiple charged particles at the Large Hadron Collider (LHC) as the test for this physics of dark matter. We discuss in Section 4 primordial nonlinear structures, reflecting the pattern of particle symmetry breaking in Axion Like Particle (ALP) models. The nonhomogeneous baryosynthesis can lead to the possibility of macroscopic antimatter sources of cosmic ray antinuclei in our Galaxy. We show in Section 4 that confirmation of the existence of such a component of cosmic rays would provide the sensitive probe for mechanisms of inflation and baryosynthesis, specifying with high precision the range of parameters of the corresponding BSM models. We then consider primordial black holes as universal theoretical probes for cosmological consequences of BSM physics (Section 5). The conclusive Section 6 puts multimessenger probes of physics of Dark Universe in the context of cosmoparticle physics, studying the fundamental relationship of micro- and macro- worlds.

\section{Cosmoparticle Physics of Particle Dark Matter}

\subsection{BSM Physics of Neutrino Mass}

The existence of primordial neutrino background is the stable prediction of the Big Bang cosmology [1,2]. Weak interaction of ordinary neutrinos keep them in equilibrium with plasma and radiation until neutrino decoupling and their number density in the modern Universe is predicted to be related to the number density of photons in Cosmic Microwave Background (CMB) radiation as

$$
n_{\nu \bar{v}}=\frac{3}{11} n_{\gamma},
$$

where $n_{v \bar{v}}$ is the number density of each type of left-handed neutrinos and right-handed antineutrinos and $n_{\gamma} \approx 400 \mathrm{~cm}^{-3}$ is the number density of CMB photons. This prediction of the Big Bang theory made neutrino historically first DM candidates, when experimental indications to the existence of nonzero mass of neutrino appeared. Multiplying the value of neutrino mass by their number density Equation (1) one obtains the modern cosmological density of massive neutrinos.

The nonzero mass of neutrino is confirmed by the experimental data on neutrino oscillations, which are determined by the difference of neutrino mass squared. The upper limit on the mass of electron neutrino puts upper limit on masses of neutrinos below $1 \mathrm{eV}$, 
what excludes the explanation of the observed dark matter density by primordial ordinary neutrino. Therefore it is not possible to explain dark matter by known particles. Moreover, the physical nature of nonzero mass of neutrino itself inevitably leads beyond the Standard model [33-36].

\subsubsection{The Nature of Neutrino Mass}

In particle theory masses of quarks and leptons are described by Dirac mass terms linking their left-handed and right-handed states. The nonzero mass of neutrino should also link the ordinary left handed neutrino to some right-handed neutrino state. On the contrary to quarks and charged leptons, right-handed neutrino is not detected-only lefthanded neutrino take part in the weak interaction. Neutrino is the only elementary matter particle, quark or lepton, which is electrically neutral. It makes possible to associate this right-handed state with ordinary antineutrino. Then neutrino has Majorana mass, which corresponds to lepton number nonconservation.

\subsubsection{The Smallness of Neutrino Mass}

Majorana nature of neutrino mass can provide the explanation, why neutrino is much lighter, than the corresponding charged lepton. This explanation involves right-handed neutrino and neutrino Dirac mass term $m_{D}$ is of the same order as for the charged lepton, but the right handed neutrino also has large Majorana mass $M \gg m_{D}$. It makes Majorana mass of ordinary neutrino suppressed, relative to the mass of the corresponding charged lepton by the factor of $m_{D} / M$ :

$$
m_{v}=m_{D} \frac{m_{D}}{M} \ll m_{D} .
$$

However, in refined models right-handed neutrino should not be necessarily much heavier, than charged leptons and their mass in $\mathrm{keV}$ range is possible [37].

\subsubsection{Sterile Neutrinos}

Right handed neutrino has no ordinary weak interaction and their interaction with SM particles is strongly suppressed. It makes them sterile relative to interactions with SM particles. If they were in equilibrium in very early Universe, their decoupling from plasma and radiation should have taken place much earlier, than for ordinary neutrinos, when much more species of relativistic particles are present in the cosmological plasma. It leads to much smaller number density of sterile neutrinos, than given by Equation (1), so that sterile neutrinos with few $\mathrm{keV}$ mass can explain the observed dark matter density [38].

The anomalous excess of electron antineutrino detected in the search for $\bar{v}_{\mu} \rightarrow \bar{v}_{e}$ oscillations by the Liquid Scintillator Neutrino Detector (LSND) [39] was considered as a possible evidence for sterile neutrinos (see Ref. [38] for recent review). Similar excess, detected in the experiment MiniBooNE at Fermilab [40] seemed to confirm the result of LSND, but the results of the successive experiment MicroBooNE did not favor such an interpretation [41]. However, these last results, excluding simple sterile neutrino interpretation of the LSND anomaly, do not exclude more sophisticated models of sterile neutrino [38,42].

\subsection{Messengers of BSM Physics of Neutrino Mass}

\subsubsection{Physics of Majorana Mass-Neutrinoless Double Beta Decay}

Majorana mass reflects nonconservation of lepton number, which should lead to processes, in which this number is not conserved.

Nuclei, which are stable relative to single beta decay can be unstable relative to double beta decay, in which two nucleons in the nucleus decay simultaneously. In the result production of nucleus in the final state is accompanied by emission of two electrons (positrons, in the case of $\beta^{+}$decay) and two antineutrinos (neutrinos). Majorana mass of neutrino makes possible convergence of a right-handed antineutrino (left handed neutrino), produced in first decay, to left-handed neutrino (right-handed antineutrino) which causes 
the second beta process. In the result neutrinoless double beta decay takes place, in which only two electrons (positrons) are emitted and there is no missed energy and momentum carried by two antineutrinos (neutrinos).

\subsubsection{Leptosynthesis}

$\mathrm{CP}$-violating effects in lepton number nonconserving decays of heavy right-handed neutrino can lead to generation of lepton asymmetry of the Universe. This process of leptosynthesis, supported by the Majorana nature of neutrino mass with lepton number transition $\Delta L=2$, leads to generation of baryon asymmetry due to electroweak sphaleron transitions at high temperature, in which baryon and lepton numbers are not conserved [43].

\subsubsection{Sterile Neutrino Dark Matter}

Sterile neutrinos can explain not only dark matter, but simultaneously generation of baryon asymmetry by leptosynthesis [44]. It can provide scenario of large-scale structure formation by warm dark matter [45]. Small mixing of sterile and active neutrino states can cause decays of sterile neutrino to photon and ordinary (active) neutrino. If sterile neutrino has mass of $7 \mathrm{keV}$, its two body decay can lead to gamma line of $3.5 \mathrm{keV}$. The claims on possible observation of radiation in such line from the galactic center [46] was discussed as a possible signature of sterile neutrino dark matter [47].

\subsection{Mirror Matter}

The extension of the Standard model by mirror partners of ordinary neutrinos, involved in the mechanisms of neutrino mass generation, may reflect the more general extension of the mirror world - of the set of mirror partners for all known particles. The existence of mirror world finds fundamental reason in the necessity to restore the equivalence of right- and left-handed coordinate systems in the conditions of $\mathrm{C}$ and P-violation in weak interactions $[48,49]$. Then existence of sterile neutrino should be accompanied by the mirror partners of quarks, leptons, W, Z bosons, as well as of the Higgs boson, while the neutrino mass becomes a narrow bridge between ordinary and mirror worlds [36,50-52]. This bridge can be extended by kinetic mixing of ordinary and mirror photon and/or other electrically neutral bosons $[29,53]$. Strict symmetry makes mirror atoms, in which mirror nuclei are bound by mirror Coulomb interaction with mirror electrons, as stable as atoms of baryonic matter.

\subsubsection{Problems of the Symmetric Mirror World}

Strict symmetry in the initial conditions and evolution of ordinary and mirror matter makes impossible to explain the observed dark matter density by mirror particles, since their density should be equal to the baryonic density. Moreover, mirror photon, electronpositron pairs and right-handed neutrinos double the number of relativistic species in the period of Big Bang Nucleosynthesis and it leads to inevitable influence on the primordial chemical composition. In particular, the primordial ${ }^{4} \mathrm{He}$ abundance is predicted in this case larger, than $28 \%$, what is excluded by the observational upper limits on the primordial helium abundance $[52,54,55]$.

\subsubsection{Asymmetric Initial Conditions for Mirror Dark Matter}

Preserving strict symmetry between ordinary and mirror particles, one can assume slightly different initial conditions for their cosmological evolution and if the temperature of mirror sector is few times smaller, than in ordinary particles, the strictly symmetric processes in mirror and ordinary worlds can lead to different results [4,56-58]. In the colder mirror sector baryosynthesis with the same physical parameters as in the ordinary sector should lead to larger mirror baryon asymmetry, while the contribution of colder mirror relativistic species in the period of Big Bang nucleosynthesis makes their existence compatible with light element abundance in the ordinary baryonic matter. On the other hand, mirror nucleosynthesis in the colder mirror plasma proceeds under the condition 
of the larger frozen out mirror neutron to proton ratio, strongly increasing the primordial mirror helium abundance.

Therefore, mirror baryonic density can be several time larger, than the ordinary baryonic density and mirror atoms and their stable constituents (mirror nuclei) can be the dominant form of dark matter, being historically the first example of physically motivated composite forms of dark matter. Formation of Large Scale structure in this scenario is triggered by the mirror matter and the scale of the structure is determined by the scale of mirror atoms recombination. It makes the corresponding scenario more close to the scenario of self-interacting and dissipative Warm Dark Matter.

\subsubsection{Shadow Matter}

Asymmetry in the physics of ordinary and mirror matter leads to shadow matter $[59,60]$, in which both particle content and the set of interactions differs from the ordinary matter. Such strong breaking of mirror symmetry takes place in the heterotic string model $E_{8} \times E_{8}^{\prime}$, in which the initial symmetry between $E_{8}$ and its mirror twin $E_{8}^{\prime}$ in 10 dimensions is broken after compactification down to $E_{6} \times E_{8}^{\prime}$ in 4 dimensions. The SM symmetry and its particle content is embedded in $E_{6}$, while the initially mirror $E_{8}^{\prime}$ describes in the four-dimensional space-time shadow world with 248 elementary particles and their 248 interactions. One can expect in this case rather nontrivial physics of dark Universe, involving multiple forms of composite dark matter particles and structures.

\subsection{Dark Matter Physics from Supersymmetry}

Supersymmetry (SUSY) puts into correspondence to each SM particle its supersymmetric partner, which has the same QCD and electroweak charge, but differs by spin [61-66]. For each SM boson is predicted its fermionic SUSY partner, while for each fermion there is a corresponding bosonic SUSY partner. Since we do not observe strict supersymmetry in the Nature, it should be broken and SUSY particles should be heavier, than their ordinary partners.

Taking apart the aesthetically attractive idea of symmetry between bosons and fermions, SUSY could provide the solution for the SM problem of divergence of Higgs boson mass, which appeared in calculations of radiative effects of virtual SM particles. In such effects virtual SUSY partners contribute with the opposite sign and the divergence of the Higgs boson mass is cancelled.

Moreover SUSY could provide explanation for the origin of the electroweak symmetry breaking scale, which is determined by the minimum of the Higgs field potential. In SUSY model this form of potential appears as the effect of renormalization of the Higgs field couplings. Such solution for both the problem of Higgs mass divergence and for the origin of the electroweak scale can be provided, if the SUSY scale and the mass of SUSY particles is in the range of hundreds GeV. It made search for SUSY particles challenging for the LHC.

\subsubsection{WIMP Miracle}

If SUSY particles possess a specific property, which ordinary particles do not possess, the lightest supersymmetric particle (LSP) should be stable and can be dark matter candidate. At the mass of hundreds $\mathrm{GeV}$ such particle has cross section of interaction with SM particles (both of annihilation and scattering) of the order of ordinary weak interaction. It makes LSP a candidate for a Weakly Interacting Massive Particle (WIMP). Frozen out in the early Universe the primordial gas of these WIMPs could be present in the modern Universe and explain the observed dark matter density.

\subsubsection{Messengers of LSP WIMP Dark Matter Physics}

SUSY Features at the LHC

Search for SUSY at the LHC was the mainstream of the collider experimental programs for the last few decades. It was motivated by the necessity in some new physical phenomena, which reflect the origin of the electroweak (EW) symmetry breaking scale. 
The discovery of Higgs boson at the LHC proved Higgs mechanism of the EW symmetry breaking, but this proof of the basic element of the SM did not provide solutions for the internal SM problems, which could be solved by SUSY and should be accompanied by the existence of supersymmetric particles.

Together with charged SUSY partners of ordinary particles, LSP production could be possible and their search by missed energy and momentum was also undertaken. However, up to now there is no evidence for SUSY physics in sub-TeV range [67].

\section{Direct WIMP Searches}

Cosmic WIMPs can freely penetrate the terrestrial matter, but with a small probability they can scatter on atomic nuclei. Such scattering on nuclei in underground detectors causes nuclear recoil, on which the principle of direct experimental WIMP search is based.

The results of this search look controversial and seem to disfavor WIMP interpretation of positive results of DAMA/NaI and DAMA/LIBRA experiments [68], which do not find confirmation in the results of direct WIMP searches by other group [69-75]. These experiments gave no evidence for WIMP-nuclear interaction and put more and more severe constraints on its parameters. Negative results are also obtained in search for light dark matter interaction with electrons in PandaX-II experiment [76].

The problem of direct comparison of results of various experiments is related with difference in their strategies and chemical composition of detectors. That is why several experiments have started to repeat all the conditions of DAMA/NaI and DAMA/LIBRA experiments, both in the chemical composition of detectors (NaI) and in strategy of search for annual modulations of dark matter signal. Taking into account that the highly statistically significant (more than $12 \sigma$ ) effect of annual modulation was detected in DAMA experiments for more than 3 decades it would be hardly possible to check this effect for a couple of years. However, the increase of sensitivity and background rejection in COSINE100 experiment makes possible to put severe constraints on WIMP interpretation of DAMA signal [77].

\section{Indirect Effects of WIMPs}

The same process of WIMP annihilation, which determined their frozen out primordial abundance in the early Universe, can take place in the Galaxy. This process involves only very small fraction of WIMPs in the Galaxy, but the SM particles, products of WIMP annihilation, contribute to the cosmic ray fluxes or gamma radiation and such excess can provide indirect search for dark matter physics [78,79]. It provides a sensitive test for existence of even subdominant component of WIMPs [80].

The excessive fraction of high-energy cosmic ray positrons registered by PAMELA and AMS02 [81-84] was considered as such an indirect effect of WIMPs. However, each source of high-energy positrons should be simultaneously the source of gamma radiation [85] and the measurements of gamma ray background by FERMI/LAT [86] put severe constraint on the dark matter interpretation of this positron excess [87].

Captured by Earth, WIMPs can annihilate inside it giving rise to the flux of neutrinos, which are accessible to neutrino telescopes. Such indirect effects can provide constrains on WIMPS in the form of scalar or Dirac neutrinos [88].

\section{Metastable Gravitino}

Local supersymmetry involves space-time and thus predicts supersymmetric partner of graviton-gravitino. Gravitino coupling contains the factor $1 / m_{P l}$ and therefore has super-weak semi-gravitational interaction. If gravitino are not the LSP, and thus are unstable, their lifetime can be still sufficiently long, being given by

$$
\tau \propto \frac{m_{P l}^{2}}{m_{G}^{3}} .
$$


Gravitino decays take place after BBN for the mass of gravitino $m_{G} \leq 10^{4} \mathrm{GeV}$. Products of gravitino decay can interact with nuclei and cause influence on light element abundance after BBN, making primordial chemical composition a sensitive probe for gravitino existence. Constraints on gravitino put restrictions on mechanisms of its production in the early Universe and correspondingly on the important features of inflationary cosmology, such as reheating temperature after inflation [89-95] or inhomogeneity of very early Universe $[29,30,32,96-98]$.

\subsection{Messengers of Supergravity}

The lack of positive evidence for SUSY particles at the LHC and for WIMPs in the underground detectors can mean that the SUSY scale is much higher, than accessible at the LHC. In the extreme case it may be at subPlanckean scale that may lead to unification of all the four fundamental forces, including gravity, on the basis of Supergravity. The models of supergravity are specified by the number of different types of gravitino $N$. The $N=1$ supergravity corresponds to the simplest case of a single gravitino.

At the SUSY super-high-energy scale gravitino has subPlanckean mass and the LSP superheavy gravitino can be the supermassive and superweakly interacting dark matter candidate in the Starobinsky supergravity, which not only explains the dark matter in this way [30,99], but also provides the implementation of the Starobinsky inflational model [13]. Extension of Starobinsky supergravity can also add the mechanism of baryosynthesis [30] putting this approach in the physical basis for the cornerstones of the modern cosmology. The price for it is the loss of the possibility to solve by supersymmetry the SM problems, which need nonsupersymmetric solutions in this case.

\section{Dark Atoms of Dark Matter}

\subsection{Multiple Charged Stable Particles}

\subsubsection{Composite Higgs and Its Charged Constituents}

The problem of divergence of the Higgs boson mass may be solved if Higgs boson is composite [100-105]. Then this mass is protected by the binding of Higgs boson constituents. The scale of this binding and its nature determines the origin of the electroweak symmetry breaking scale. Higgs boson is neutral, but its constituents can be electrically charged and can form some other bound states with nonzero electric charge [106,107]. If stable, they can be bound in neutral "dark atoms", which can play the role of dark matter.

\subsubsection{Problem of Anomalous Isotopes}

The main problem for existence of stable charged particles is a possible overproduction of anomalous isotopes. Created in the early Universe, they can hardly recombine completely in dark atoms in the course of cosmological evolution and even a very small fraction of free +1 charged particles bound with electrons leads to overproduction of anomalous hydrogen which is severely constrained by the experimental data. The similar problem appears for any free particles with positive integer charge, $Z>1$ which can be bound with electrons in the anomalous isotope of the corresponding element $Z$. If stable particles have fractional charge, their abundance is severely constrained by the experimental searches for free quarks. Free particles with negative odd charge $-(2 n-1)$ (where $n=1,2, \ldots)$ are captured by $n$ nuclei of primordial helium, as soon as it is produced in the Big Bang Nucleosynthesis, forming +1 charged ions that play the same dangerous role of 'nuclei' of anomalous hydrogen.

However, there does not appear such an evident contradiction for charged particles with negative charge $-2 n$, which can be bound with $n$ nuclei of primordial helium in neutral $\mathrm{OHe}(n=1)$ or XHe dark atoms [28,29].

\subsubsection{Dark Atom Constituents}

Stable particles with nonzero electric charge are predicted: 
(a) as AC-leptons in the extension of the Standard Model, based on the approach of almost-commutative (AC) geometry $[85,108,109]$.

(b) as technileptons and anti-technibaryons in the framework of Walking Technicolor (WTC) [110-116].

The absolute value of electric charge is not fixed in these models and the relative charge assignment is determined by the compensation of anomalies and the condition of electroneutrality. These particles have no QCD interaction and thus behave as heavy stable multiple charged leptons in the interaction with SM particles.

Another possibility appears in the case of stable "heavy quark clusters" $\bar{U} \bar{U} \bar{U}$ formed by stable anti- $U$ quark of 4 th generation [117] or stable charged clusters $\bar{u}_{5} \bar{u}_{5} \bar{u}_{5}$ of stable (anti)quarks $\bar{u}_{5}$ of 5 th family, which can be predicted in the approach, unifying spins and charges [118]. The precise measurements of Higgs boson properties put severe constraints on the deviations from the predictions of the Standard model. To satisfy these constraints sophisticated WTC scenarios (see [119] for recent review) or strong suppression of heavy quark coupling to $125 \mathrm{GeV}$ Higgs boson [120] are needed [28,29,85].

\subsubsection{Suppression of Anomalous Isotopes}

All the negatively charged particles are inevitably accompanied by the corresponding positively charged antiparticles, which can recombine with ordinary electrons in anomalous isotopes. The experimental constraints on anomalous isotopes of elements with $Z \geq 2$ are not as strong as for anomalous hydrogen but still can cause a problem for the considered scenario. There are two possible mechanisms that can provide a suppression of anomalous isotopes in the terrestrial matter:

(i) The abundance of anomalous isotope in the Galaxy may be significant, but in terrestrial matter recombination of negatively charged particles with positively charged particles suppresses this abundance below experimental upper limits [108]. This mechanisms implemented in the scenario with AC leptons, forming neutral AC atoms [108], implies a new $\mathrm{U}(1)$ gauge symmetry, causing new Coulomb-like long range interaction between $\mathrm{A}$ and $\mathrm{C}$ leptons, having opposite electric charge. It leads inevitably to the existence of dark radiation in the form of hidden photons mediating the $\mathrm{U}(1)$ interaction between $\mathrm{A}$ and $\mathrm{C}$ lepton [85].

(ii) If new stable particles are in non-trivial representations of the $\mathrm{SU}(2)$ electroweak group, sphaleron transitions at high temperatures establish balance between baryon asymmetry and the excess of these particles. Such excess of negatively charged stable particles was shown to appear naturally in the case of WTC $[110,121]$ and to provide suppression of positively charged antiparticles. This charge asymmetric solution maintains relationship between densities of asymmetric dark matter and baryonic matter and is in the basis of dark atom cosmology.

\subsection{Dark Atom Cosmology and Its Probes}

\subsubsection{Dark Atoms}

Dark atom cosmology assumes that the excessive $-2 n$ charged particles bind with $n$ nuclei of He, as soon as it is created in BBN. Scenario of dark atom Universe involves only one parameter of new physics-the mass of $\mathrm{O}^{--}$or $\mathrm{X}^{--}$and the main features of the dark atom interaction with matter are determined by their nuclear interacting (multi-)helium shell.

The main problem of proper treatment of dark atom interaction with matter is related with the lack of usual approximations, typical for atomic physics.

Dark atom is an $\mathrm{OHe}$ Bohr atom, in which heavy double charged heavy lepton $\mathrm{O}^{--}$is surrounded by helium shell with Bohr orbit equal to the size of helium nucleus, or Thomson $X$ He atom in which $-2 n$ charged heavy lepton $X$ is situated within the nuclear droplet of $n$ He nuclei $[28,122-124]$.

Therefore, though dark atom scenario can be in principle based on known atomic and nuclear physics, specifics of dark atom structure makes the problem very complicated as 
compared with the case of ordinary atoms, in which electronic shells have Bohr radius, which is much larger, than the size of strongly interacting nucleus. The small parameters of electromagnetic interaction of shells and of the ratio of nucleus to shell radii provide the usual approximations of atomic physics, which are not the case for dark atoms. Dark atom interaction with nuclei involves a self-consistent treatment of the simultaneous action of nuclear attraction and Coulomb repulsion between He shell (or droplet) and approaching nucleus under the condition that the finite size of nuclei should be taken into account.

Qualitatively, the following picture may be expected [85]. At large distances Coulomb field of nucleus polarizes dark atom so that Stark-effect attraction takes place. At some distance nuclear attraction changes the sign of polarization, but Coulomb repulsion moves it back, and then nuclear attraction moves $\mathrm{He}$ (in case of $\mathrm{OHe}$, or nuclear droplet in case of $\mathrm{XHe}$ ) again towards nucleus, and so on. The mutual effect of nuclear attraction and Coulomb repulsion can lead to a low energy bound state of dark atom and intermediate mass nuclei. The existence of such state can explain the puzzle of direct dark matter searches and this approach is now being developed for quantitative investigation $[125,126]$. Coulomb repulsion between the nucleus and nuclear content of dark atom can provide dominance of elastic scattering of dark atoms off nuclei, on which the qualitative features of dark atom cosmology are based [127,128].

\subsubsection{Dark Atom Scenario}

Elastic nuclear interactions of dark atom helium constituent with cosmic plasma nuclei held dark atoms in thermal equilibrium with plasma and radiation on the Radiation Dominance (RD) stage, while the energy and momentum transfer from plasma is effective. It converted dark atom density fluctuations in acoustic waves at scales up to the size of the horizon and lead to slight suppression of the small scale fluctuations, which can not be as strong as the free streaming suppression in ordinary Warm Dark Matter (WDM) scenarios. As soon as the interaction with plasma becomes ineffective, dark atom gas decouples from plasma and radiation and plays the role of Warmer than Cold dark matter [28,85].

The dark atom gas does not follow the formation of baryonic astrophysical objects until their size $R$ and number density $n_{m}$ satisfy the condition $n_{m} \sigma R<1$, where $\sigma \approx 210^{-25} \mathrm{~cm}^{2}$ is the cross section of dark atom interaction. Dark atoms form dark matter halos of galaxies and behave as collisionless gas at the galactic scales [85]. They can be captured by dense baryonic objects like stars or planets.

\subsubsection{Dark Atom Explanation for Puzzles of Direct DM Searches}

Cosmic dark atoms from galactic halo are slowed down in the terrestrial matter. It makes them elusive for direct WIMP searches, in which detection is based on nuclear recoil from WIMP scattering [85]. The positive results of DAMA/NaI and DAMA/LIBRA experiments (see [68] for review and references) find in this scenario a nontrivial explanation based on a low energy binding of dark atom with intermediate mass nuclei [25].

Local dark atom concentration is determined in the terrestrial matter by the equilibrium between incoming flux of cosmic dark atoms and their diffusion towards the center of Earth. At the level of underground detectors the adjustment to the incoming flux takes about an hour. The incoming cosmic flux experiences annual modulation due to the Earth's orbital motion. It leads to annual modulation in the local concentration of dark atoms in the underground detector. Then radiative capture of dark atoms by nuclei in DAMA detector possess annual modulation and it explains the positive result of DAMA/NaI and DAMA/LIBRA experiments.

A low energy binding of dark atoms with nuclei is crucial for this explanation. Such binding was found for intermediate mass nuclei in the approximation of square well and wall for $\mathrm{OHe}[25,85]$, and it resulted in the existence of a few keV level in the $\mathrm{OHe}-\mathrm{Na}$ system. The positive result of DAMA/NaI and DAMA/LIBRA experiments follows then from annual modulation of the rate of $\mathrm{Na}$ capture by $\mathrm{OHe}$ to the $3 \mathrm{keV}$ bound state of OHe-Na system. 
In the same approximation it was shown that such level does not exist in OHe interaction with light and heavy nuclei. In particular, there is no such level in OHe interaction with xenon and it can explain negative results of the XENON100 and LUX experiments.

Owing to the scalar nature of He nucleus radiative transition to the bound state of OHe with intermediate mass nuclei can go only by the electric dipole transition, which makes the corresponding rate proportional to the temperature, and thus suppressed in cryogenic detectors.

Selection rules imply isovector character of nuclear electric dipole transition and it involves isospin violation in the case of isoscalar He nucleus. The account for the corresponding suppression factor made possible to reproduce the number of events, detected in DAMA/NaI and DAMA/LIBRA experiments [25,85].

\subsection{Indirect Effects of Dark Atoms}

\subsubsection{O-Nuclearites}

Heavy nuclei can bind with multiple $\mathrm{O}^{--}$particles and form exotic $O$-nuclearites, neutral superheavy nuclear states, in which electric charge of nucleus is compensated by the negative charge of $\mathrm{O}^{--}$particles $[85,129]$. In the lack of heavy nuclei in the early Universe $O$-nuclearites can hardly have primordial origin but they can be formed in stars due to O-helium capture. $\mathrm{O}$-nuclearites, created in stars, can be accelerated in the shock waves at supernova explosions and form anomalous component of cosmic rays. Capture of a sufficiently large amount of $\mathrm{OHe}$ can trigger collapse of neutron star to black hole [130]. It can put some constraints on the properties of $\mathrm{O}^{--}$particles $[85,129]$.

\subsubsection{Exotic Component of Cosmic Rays}

In addition to $\mathrm{O}$-nuclearites dark atoms can give rise to some other exotic forms of cosmic rays. High energy cosmic rays can scatter on them and set free their multiple charged constituents. Supernova explosions of the I type, which leave no compact remnant, release all the dark atoms, captured by star in the course of its evolution. Ionization in the front of the shock wave releases free $O$ or $X$ constituents, which can be accelerated and become a multiple charge lepton component of cosmic rays $[85,117]$. The main problem in confrontation of these predictions with the results of cosmic ray experiments is whether the mechanisms of acceleration are sufficiently effective to provide detectable fluxes.

Positron Annihilation in the Center of Galaxy

Though dark atoms behave like collisionless gas, the rate of their collisions depends on the dark atom number density $n$ and grows with $n$ as $n^{2}$. It can make effect of such collisions observable in the central part of the Galaxy, where dark atom density is maximal. Dark atoms can be excited in their collisions. Excitation to $2 S$ level leads pair production in E0 transition of de-excitation, while de-excitations from other excited levels lead to specific series of gamma lines. Pair production in dark atom de-excitations can explain the excess of the positron-annihilation line, observed in the galactic bulge by INTEGRAL $[25,85,121,131-133]$. Such explanation implies the mass of double charged constituent of O-helium to be in a narrow window around [85]

$$
m_{o}=1.25 \mathrm{TeV} \text {. }
$$

\subsubsection{Decaying Dark Atom Constituent}

Pending on the conservation of technibaryon and technilepton numbers WTC model can predict stable technilepton $\mathrm{O}^{--}$and a metastable $\mathrm{U}^{++}$technibaryon. The latter has the lifetime exceeding the age of the Universe $[85,134]$. It leads to a dark atom model, in which the dominant nuclear-interacting component of OHe dark atom is accompanied by a sub-dominant WIMP-like component $(O U) . U^{++}$decays to pairs of positive-sign leptons can provide explanation for the excessive high-energy positrons [85], detected in cosmic-rays by PAMELA and AMS02 experiments [81-84]. Such explanation should avoid 
overproduction of gamma background, which inevitable accompanies these decays. It puts the following upper limit on the mass $m_{u}$ of the decaying constituent of dark atom [85]

$$
m_{u}<1 \mathrm{TeV} \text {. }
$$

These predictions provide their test in experimental search for stable multiple charged particles.

\subsection{Experimental Search for Dark Atom Constituents}

\subsubsection{Searches for Stable Multiple Charged Particles}

Searches for exotic stable particles with multiple electric charge are continuously carried out in cosmic ray and collider experiments (see, e.g., for review [135]). Conservation of electric charge forbids in a tree approximation decay of double charge particles to pair of quarks so that only decays to the leptons of the same sign are possible. Such process implies lepton number nonconservation and can be a signature of BSM physics. It would make multiple charged particles of any origin sufficiently long living.

In the context of dark atom hypothesis search for stable multiple charged heavy leptons acquires the meaning of test for the atom-like structure of the cosmological dark matter. Moreover, since dark atom model can explain the excess in positron annihilation line and high-energy positron fraction in cosmic rays only if the mass of double charged $\mathrm{O}^{--}$particles is in the $1 \mathrm{TeV}$ range, search for such particles in this range is the experimentum crucis for such explanation.

\subsubsection{Search for Multiple Charged Particles at the LHC}

No events with multiple charged particles were found in ATLAS experimental data sets, setting the lower mass limits in the $1 \mathrm{TeV}$ at 95\% CL [136] as shown in Figure 2. The observed cross-section upper limits are compared in Figure 2 with theoretically predicted cross-sections.

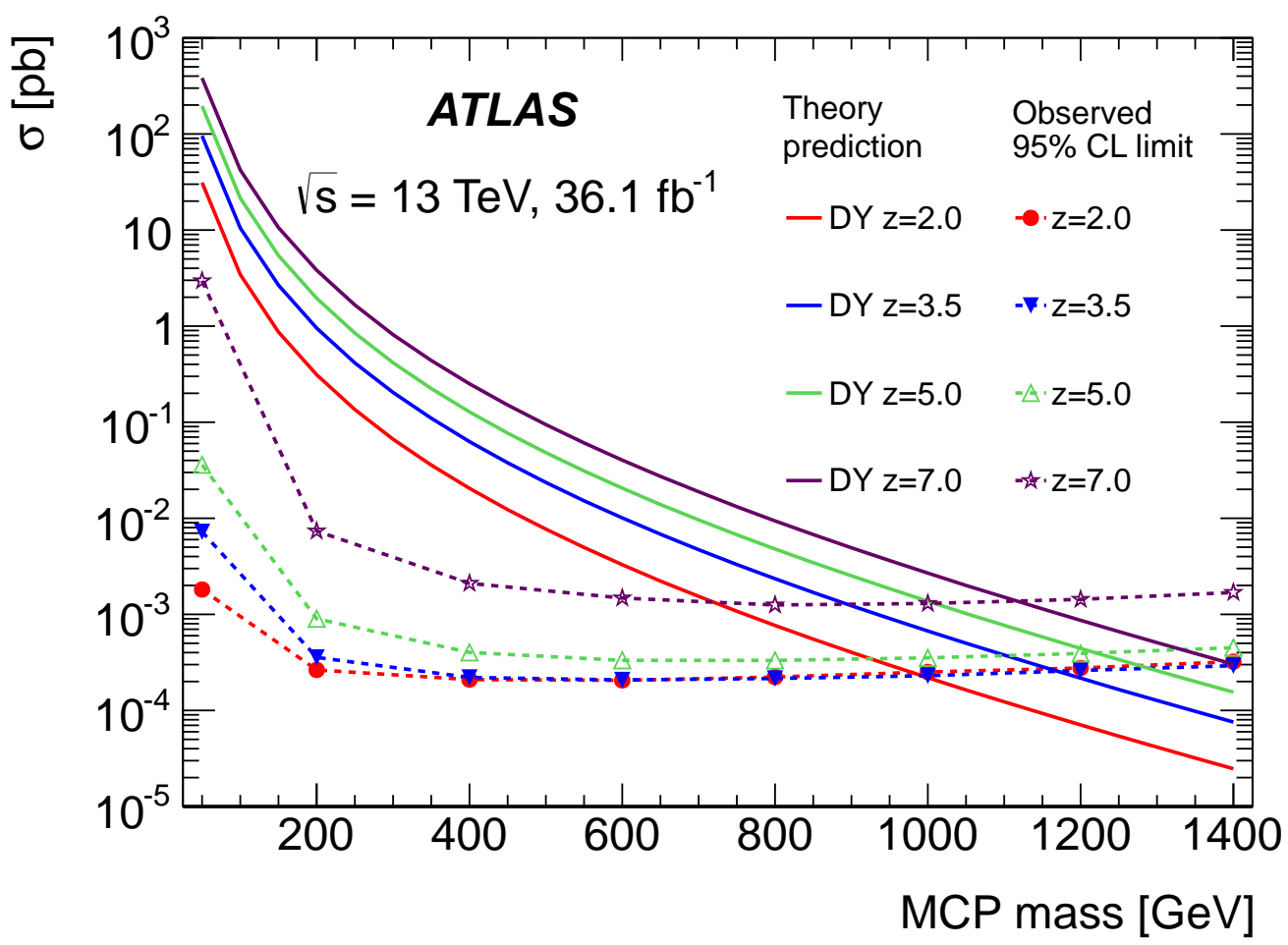

Figure 2. Observed 95\% CL cross-section upper limits and theoretical cross-sections as functions of the multi-charged particles mass [136]. The double charged particles are denoted as " $z=2$ " (red points and lines). The author's original picture from [134]. 
It was concluded in [134] that the results of CMS and ATLAS experiments are able to set a lower mass limit on the double charged particles at $m=1000 \pm 50 \mathrm{GeV}$. It already excludes interpretation of the excess of high-energy cosmic positrons in terms of dark atoms with decaying $U^{++}$double charged constituents.

The further analysis of ATLAS and CMS experimental data was estimated in [134] to cover all the range of masses for stable double charged particles, at which the observed excess of low and high-energy positrons can be explained as a possible indirect effect of dark atoms with such constituents. A possibility of such explanation in the case of multiple charged constituents of XHe dark atoms in confrontation with search for these constituents in cosmic rays and at the LHC needs special study.

\section{Primordial Structures from BSM Physics}

The pattern of particle symmetry breaking determines the succession of phase transitions in the early Universe. Pending on the pattern of symmetry breaking topological defects, like magnetic monopoles, cosmic strings or domain walls can be formed in such transitions [137-145]. If stable, such defects can provide sensitive cosmological probe for the corresponding model [29]. However, even temporary existence of such defects, which rise and then disappear in the succession of phase transitions can lead to observable effect, as we discuss on the example of Axion-Like-Particle (ALP) models.

\subsection{Cosmological Structures from Axion-like Models}

The problem of divergence of Higgs boson mass in the electroweak sector of SM is accompanied by the problem of strong CP violation in QCD, revealed by R. Peccei and H. Quinn [146], who proposed additional global U(1) symmetry, which provided solution for this problem. However, similar to the case of SUSY, which predicts existence of supersymmetric partners of ordinary particles, spontaneous breaking of Peccei-Quinn (PQ) symmetry involves prediction of a pseudo-Nambu-Goldstone boson-axion [147,148]. Constraints on this QCD axion imply rather high-energy scale of the spontaneous PQ symmetry breaking, making axion invisible, but potentially very attractive as candidate for cosmological dark matter (see Refs. $[149,150]$ for review and references). Extension of axion models leads to development of models of axion-like particles, which are not related with the PQ solution for the QCD problem.

A wide class of axion-like particle (ALP) models can be effectively described by a complex field, which experiences a succession of spontaneous and then explicit breaking of global U(1) symmetry. Spontaneous U(1) symmetry breaking is induced by the vacuum expectation value $\langle\psi\rangle=f$ of a complex scalar field $\Psi=\psi \exp (i \theta)$. The explicit symmetry breaking is provided by the term in its potential $V_{e b}=\Lambda^{4}(1-\cos \theta)$. Spontaneous symmetry breaking at scale $f$ results in continuous degeneracy of vacua. The explicit symmetry breaking at smaller energy scale $\Lambda \ll f$ changes this continuous degeneracy by discrete vacuum degeneracy. The character of formed structures depends on whether the first phase transition with spontaneous symmetry breaking takes place in the inflationary or post-inflationary stage.

\subsubsection{Large Scale Correlations of Axion Field}

If the first phase transition proceeds at the high temperature after reheating, due to continuous degeneracy of vacua a string network is formed. At the second phase transition, the initial phase value, acquires a dynamical meaning of the amplitude of the ALP field, when ALP mass is switched on Then the string network transforms in the topological defect structure of walls surrounded by strings. This defect structure is unstable, but it leaves a replica in the energy density distribution of coherent ALP field oscillations [151-153], reflected in the large-scale inhomogeneity of this distribution.

The value of phase changes by $2 \pi$ around string. The common wisdom (see References [154,155] and references therein) is that the corresponding nonhomogeneity is not essential at the distances, exceeding the size of the cosmological horizon in the period 
when ALP field oscillations start. However, since the nonhomogeneity of phase follows the pattern of the wall-surrounded by strings network, this general wisdom misses large-scale correlations in the energy density distribution of ALP oscillations.

One may think that such correlations can not survive at large distances, if large loops are dominant in them, but the numerical analysis (see the review in [156]) indicates that large loop contribution is strongly suppressed, and the dominant fraction of 'infinite' strings (about $80 \%$ of string length) is virtually constant in all large scales. This property reflects the scale invariant character of the cosmic string network. Therefore, the large-scale correlations of energy density distribution should be present in ALP cosmology, if the first phase transition took place after reheating, as it was revealed in References [151-153]. Cosmological evolution of these structures and their observational effects need special study. If these large-scale correlations persist to the period large-scale structure formation, their contribution into the $\mathrm{CMB}$ anisotropy put severe constraints on the possible fraction of dark matter in the form of ALP and in particular can exclude axions as the dominant form of Cold Dark Matter [151-153].

Formation of large scale primordial inhomogeneities embracing causally disconnected regions in the period of phase transitions is the consequence of inflation, which provides the identical conditions of phase transition after reheating in these regions, supporting large-scale correlation of inhomogeneities, formed in them.

\subsubsection{Primordial Seeds for Active Galactic Nuclei}

If the phase transition with spontaneous $\mathrm{U}(1)$ symmetry breaking take place at the inflational stage, the value of $\theta_{60}$ is fixed in the period of inflation with $e$-folding $N=60$ corresponding to the part of the universe within the modern cosmological horizon. At successive steps of inflation the phase $\theta$ experiences fluctuations of the order of $\Delta \theta \sim$ $H /(2 \pi f)$ (here $H$ is the Hubble parameter at the inflational stage) [157]. They change in the course of inflation, the initial value of phase within the regions of smaller size. If $\theta_{60}<\pi$, the fluctuations can move at $N<60$ the value of $\theta_{N}$ to $\theta_{N}>\pi$ in some regions of the universe. After reheating, when the universe cools down to temperature $T=\Lambda$, the explicit symmetry breaking leads to the phase transition to the discrete vacuum states. They correspond to the minima of $V_{e b}$. For $\theta_{N}<\pi$, the minimum of $V_{e b}$ is reached at $\theta_{v a c}=0$; while in the regions with $\theta_{N}>\pi$, the vacuum state corresponds to $\theta_{v a c}=2 \pi$.

If $\theta_{60}<\pi$ the true vacuum state within the modern cosmological horizon corresponds to $\theta_{\text {vac }}=0$. However, owing to phase fluctuations within this volume there can appear compact regions with $\theta_{\text {vac }}=2 \pi$. These regions are separated from the bulk by massive domain walls, which are formed at the border between the two vacua. Since regions with $\theta_{\text {vac }}=2 \pi$ are compact, the domain walls are closed. After these closed walls enter the horizon, they can collapse into black holes (BHs).

The mass range of formed BHs is determined by the scales of symmetry breaking, $f$ and $\Lambda$. The condition that the wall does not dominate locally before it enters the cosmological horizon defines the principally maximal mass of black hole. Otherwise, local wall dominance leads to a superluminal $a \propto t^{2}$ expansion of the corresponding region, making it elusive for the other part of the universe. This condition corresponds to the mass [158]

$$
M_{\max }=\frac{m_{p l}}{f} m_{p l}\left(\frac{m_{p l}}{\Lambda}\right)^{2}
$$

The minimal mass is determined by the condition that the gravitational radius of $\mathrm{BH}$ exceeds the width of wall. It is equal to $[158,159]$

$$
M_{\text {min }}=f\left(\frac{m_{p l}}{\Lambda}\right)^{2}
$$

Closed wall collapse leads to primordial Gravitational Wave (GW) background, peaked at [29]

$$
v_{0}=3 \times 10^{11}(\Lambda / f) \mathrm{Hz}
$$


with energy density up to

$$
\Omega_{G W} \approx 10^{-4}\left(f / m_{p l}\right)
$$

At $f \sim 10^{14} \mathrm{GeV}$ this primordial GW background can reach $\Omega_{\mathrm{GW}} \approx 10^{-9}$. For the physically reasonable values of

$$
1<\Lambda<10^{8} \mathrm{GeV}
$$

the maximum of the spectrum corresponds to

$$
3 \times 10^{-3}<v_{0}<3 \times 10^{5} \mathrm{~Hz}
$$

In the range from tens to thousands of $\mathrm{Hz}$, such background may be a challenge for the gravitational wave experimental search.

The first crossing of $\pi$ by phase fluctuation at some stage of inflation $N_{1}$ is accompanied by series of its $i$ (where $i$ is a natural number) successive crossings at $N(i)<N_{1}$. Therefore formation of a larger closed wall is accompanied in the corresponding region by formation of $i$ smaller walls. It naturally leads to formation of primordial black hole (PBH) clusters [160]. Gravitational wave signals from merging of BHs in such cluster is another profound signature of the considered scenario, which may be tested in directional detection of correlation of $\mathrm{BH}$ merging in $\mathrm{GW}$ experiments.

In principle, the mechanism of closed wall collapse can lead to the formation of primordial black holes of a whatever large mass up to the mass of seeds of active galactic nuclei (AGNs) [161,162], see for review References [31,160]. Such black holes appear in the form of primordial black hole clusters, exhibiting fractal distribution in space $[158,159,163]$. This can shed new light on the problem of galaxy formation [158,162].

The prediction of massive PBHs and their clustering can provide the interpretation of GW signals from massive black hole merging. Continuously growing Catalogs of GW signals [164-166] contain dominantly the detected signals from the coalescence of black hole binaries (BBH) with the measured mass larger than 10-20 $M_{\odot}$. Formation of such massive black holes is difficult to explain by the astrophysical models of the evolution of the first stars, especially if the mass exceeds the "pair instability" threshold about $50 \mathrm{M}_{\odot}$. The primordial origin of massive and supermassive black holes may resolve this difficulty (see, e.g., [167] for review and references), as well as massive PBH clustering can facilitate BBH formation [168]. In that context, a GW signal from a BBH coalescence with total mass $150 M_{\odot}$, detected by LIGO and VIRGO collaborations [169], was recently considered as possible evidence for primordial origin of massive BHs [170].

Clustering of massive PBHs, lead to repeating events of BBH coalescence in the cluster, and can provide an observational test for for PBH clusters [160,171]. If confirmed, it will strongly favor BSM models, predicting the formation of massive PBH clusters in the early Universe, as well as specify their parameters.

\subsection{Macroscopic Antimatter from BSM Physics}

Strong inhomogeneity of baryosynthesis can lead to the appearance of antibaryon domains in the baryon asymmetrical universe [172]. In an example of a model of spontaneous baryosynthesis (see Reference [173] for review), it was shown in [174] how the combination of inflation and nonhomogeneous baryosynthesis can result in the formation of sufficiently large antimatter domains, which can survive to the present time.

Antimatter Domains in Baryon Asymmetrical Universe

The mechanism of spontaneous baryogenesis $[173,175,176]$ implies the existence of a complex scalar field $\chi=(f / \sqrt{2}) \exp (\theta)$ carrying the baryonic charge. Similar to the case of ALP, the global $U(1)$ symmetry of the baryon charge is broken spontaneously and explicitly. The explicit breakdown of $U(1)$ symmetry is caused by the phase-dependent term

$$
V(\theta)=\Lambda^{4}(1-\cos \theta)
$$


The interaction of the field $\chi$ with matter fields violates baryon and lepton number [173]:

$$
\mathcal{L}=g \chi \bar{Q} L+\text { h.c., }
$$

where $Q$ and $L$ are, respectively heavy quark and lepton fields, which are coupled to the ordinary matter fields.

When in the course of post inflational stage the slows down and becomes comparable with the mass $m_{\theta}=\frac{\Lambda^{2}}{f}$, the phase $\theta$ acquires dynamical meaning and starts to oscillate around the minimum of its potential (12), decaying to matter fields. The interaction (13) leads the following feature of the decay products [173]: it creates the baryon excess, when the phase starts to roll down in the clockwise direction, and antibaryon excess, if it starts to roll down in the anti-clock-wise direction. Therefore the excess of baryons or antibaryon is determined by the initial value of $\theta$, fixed in the inflational stage.

This specifics of baryon and antibaryon excess generation is illustrated on Figure 3. If the value of phase $\theta_{60}$ at the e-fold $N=60$, corresponding to the observed part of the modern Universe, is in the range $[\pi, 0]$, there is created the net excess of baryons. However at the successive stages of inflation, at each successive e-fold, corresponding to smaller scales, this value experiences Brownian steps $\delta \theta=H_{\text {infl }} /(2 \pi f)$, where $H_{\text {infl }}$ is the Hubble parameter at the inflational stage. The typical scale of the fluctuation $\delta \theta$ is equal to $H_{\text {infl }}^{-1}$. The whole domain $H_{\text {infl }}^{-1}$ containing phase $\theta_{N}$ gets divided (after one e-fold) into $e^{3}$ causally disconnected domains of radius $H_{\text {infl }}^{-1}$. Each new domain contains almost homogeneous phase value $\theta_{N-1}=\theta_{N} \pm \delta \theta$. This process repeats in every domain with every successive e-fold and at some step $N$ the phase $\theta_{N}$ can cross the value of $\pi$. In this domain the direction of rolling down to the minimum of potential (12) is anti-clockwise and antibaryon excess is generated in this region, being surrounded by baryon excess outside it.

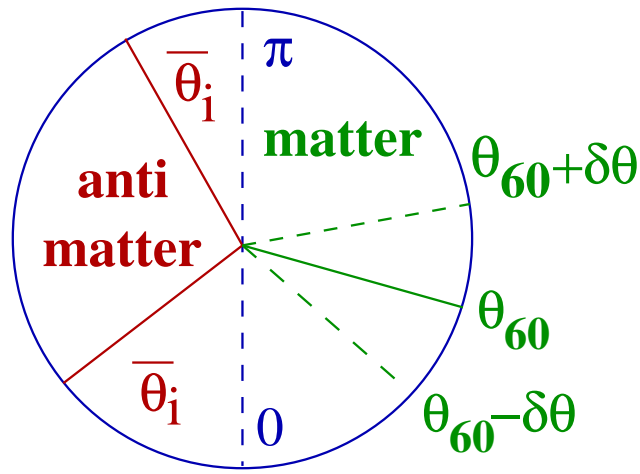

Figure 3. The evolution of the phase at the inflational stage (the author's original picture from Reference [177]).

The fate of such antimatter regions depends on their size and antibaryon density within them. If their size exceeds the critical surviving scale $L_{c}=8 h^{2} \mathrm{kpc}$ [174], diffusion to the borders of domain cannot lead to their complete dissipation and they survive annihilation with surrounding matter. Low density domains can survive in the form of diffused antimatter regions, in which antibaryon density is so low that neither nucleosynthesis, nor recombination is possible in them, so that they represent the regions of low density antiproton-positron plasma [174].

Sufficiently dense antimatter domains can evolve in antibaryon objects, which can be present in our Galaxy. If evolution of antibaryon matter in them was similar to the evolution of baryon matter, antimatter globular cluster can be formed in our Galaxy [178]. The existence of such cluster in the halo of our Galaxy cannot lead to strong effect of antimatter annihilation within it due to low density of matter gas in halo and to the fact that annihilation can be only on the surface of antimatter stars, making antimatter globular cluster rather faint gamma ray source. More stronger effect comes from the pollution of the 
galactic halo by antinuclei (dominantly antiprotons) lost by antimatter stars by anti-stellar winds. The annihilation of such antinuclei with matter gas can contribute to [179] the observed galactic gamma background in the range of tens-hundreds $\mathrm{MeV}$, which puts an upper limit on the total mass of antimatter stars about $10^{5} \mathrm{M}_{\odot}$ [178]. Rough estimation of the predicted antihelium component of cosmic rays [180] makes it accessible to searches for cosmic ray antinuclei in AMS02 experiment and if the presence of such component is confirmed, it can be hardly explained as secondary antinuclei [181].

One can think that antimatter meteorites [182] can also provide a direct experimental test for the hypothesis of macroscopic antimatter in our Galaxy. However, formation of such meteorites assumes existence of antimatter dust, which can be hardly formed in the conditions of antimatter localized within a region of antimatter globular cluster. The elementary constituents (anti-carbon, anti-silicon etc.) of such dust cannot come to this region from surrounding matter, while being formed within the globular cluster are easily lost and annihilated in the interstellar matter gas.

If $\theta_{60} \ll \pi$ and fluctuations move the value of phase to $\theta>\pi$, antibaryon density in antimatter domain may be much higher, than in baryonic matter. It can result in formation of very dense self-gravitating antibaryon regions, whose evolution can differ substantially from ordinary baryonic matter and result in formation of superdense antibaryonic objects in our Galaxy. The possibility of the formation of such dense antimatter objects and the strategies for their search were considered in [183].

To conclude, the primordial strong inhomogeneities enrich the variety of cosmological messengers the Dark universe. Primordial black holes play special role in the list of such cosmological messengers and we consider in the next section the variety of $\mathrm{PBH}$ probes for symmetry breaking pattern of physics of dark Universe.

\section{Primordial Black Holes as a Probe for Physics of Dark Universe}

Gravitational collapse of mass $M$ within its gravitational radius $r_{g}=2 G M / c^{2}$. can naturally take place in the end of evolution of star, whose mass exceeds three solar mass $[184,185]$. However, in the early universe, as noticed Ya. B. Zeldovich and I. D. Novikov (see Reference [186]), if cosmological expansion stops in some region within the cosmological horizon, a black hole of a sub-stellar mass can be formed. This implies strong deviation from general expansion and inhomogeneity leading to formation of Primordial Black Holes (PBHs) $[187,188]$. Such strong inhomogeneities reflect various features of BSM physics at superhigh energy scale [31].

\subsection{PBH Probe for Superheavy Metastable Particles}

In the universe with equation of state

$$
p=\gamma \epsilon
$$

with numerical factor $\gamma$ being in the range

$$
0 \leq \gamma \leq 1
$$

the probability of forming a black hole from fluctuations with dispersion

$$
\left\langle\delta^{2}\right\rangle \ll 1
$$

within the cosmological horizon is given by [189]

$$
W_{P B H} \propto \exp \left(-\frac{\gamma^{2}}{2\left\langle\delta^{2}\right\rangle}\right) .
$$


It makes PBH spectrum exponentially sensitive to the softening of the equation of state $(\gamma \rightarrow 0)$ or to the enhanced ultraviolet part of the spectrum of fluctuations $\left(\left\langle\delta^{2}\right\rangle \rightarrow 1\right)$. These phenomena can reflect cosmological consequence of BSM physics [31].

\subsubsection{PBHs from Dominance of Superheavy Particles}

The existence of superheavy metastable particles with lifetime $\tau \ll 1 s$ cannot be directly probed by in the astrophysical. However, it was first noticed in Reference [190] that if such particles dominate in the universe before their decay at $t \leq \tau$ they can form PBHs, which keep information on particle properties in their spectrum. This provides an indirect possibility to probe the existence of such particles by confrontation of the effect of the predicted PBH spectrum with astrophysical observations.

After reheating, at

$$
T<T_{0}=r m
$$

particles with mass $m$ and relative abundance $r=n / n_{r}$ (where $n$ and $n_{r}$ are correspondingly number densities of particles and of relativistic species) dominate in the universe before their decay. At matter dominance of these nonrelativistic particles at $t>t_{0}$, where

$$
t_{0}=\frac{m_{p l}}{T_{0}^{2}}
$$

density fluctuations grow as

$$
\delta(t)=\frac{\delta \rho}{\rho} \propto t^{2 / 3} .
$$

In the result of the development of gravitational instability gravitationally bound systems are formed, which decouple at

$$
t \sim t_{f} \approx t_{i} \delta\left(t_{i}\right)^{-3 / 2}
$$

from general cosmological expansion, when fluctuations entering the horizon with amplitude $\delta\left(t_{i}\right)$ at $t=t_{i}>t_{0}$ grow to $\delta\left(t_{f}\right) \sim 1$.

Black holes can form either directly after the system decouples from expansion or in the result of evolution of the initially formed gravitationally bound systems.

\subsubsection{Direct PBH Formation}

Density fluctuation can directly collapse to $\mathrm{BH}$ if it is especially homogeneous and isotropic, so that it contract within its gravitational radius, when the system decouples from expansion. A probability for such direct collapse gives minimal estimation of $\mathrm{BH}$ formation in the matter dominated stage.

This probability was calculated in Reference [190] for configuration with such a high sphericity and homogeneity that they can contract within their size, which they had, when entered horizon and which corresponds to their gravitational radius. The corresponding conditions are independent of the specific properties of dominating matter, being valid for both collisionless and interacting particles [191-194],

The probability of sufficient sphericity is given by [190-194]

$$
W_{s} \sim \delta\left(t_{i}\right)^{5},
$$

where $\delta\left(t_{i}\right)$ is the amplitude of fluctuation, when it entered horizon at $t-t_{i}$-the product of this probability and the probability for sufficient homogeneity

$$
W_{u} \sim \delta\left(t_{i}\right)^{3 / 2}
$$

leads to the strong power-law suppression of probability for direct BH formation

$$
W_{P B H}=W_{s} \cdot W_{u} \sim \delta\left(t_{i}\right)^{13 / 2}
$$


This calculation [190-194] does not imply the specific form of Gaussian distribution of fluctuations and thus should not change strongly in a case of non-Gaussian fluctuations [195].

This mechanism [3,4,190-194] leads to formation of PBHs with mass in an interval

$$
M_{0} \leq M \leq M_{\text {bhmax }}
$$

The minimal mass is given by the mass within the cosmological horizon in the beginning of matter dominance at $t \sim t_{0}$, and is equal to $[3,4,190-194]$

$$
M_{0}=\frac{4 \pi}{3} \rho t_{0}^{3} \approx m_{p l}\left(\frac{m_{p l}}{r m}\right)^{2}
$$

The maximal mass is follows from the condition

$$
\tau=t\left(M_{\text {bhmax }}\right) \delta\left(M_{\text {bhmax }}\right)^{-3 / 2}
$$

that fluctuation with the mass $M_{\text {bhmax }}$, which enters horizon at $t\left(M_{\text {bhmax }}\right)$ with an amplitude $\delta\left(M_{\text {bhmax }}\right) \ll 1$, can collapse before particles decay at $t=\tau$. For scale-invariant spectrum $\delta(M)=\delta_{0}$, the maximal mass is given by [158]

$$
M_{\text {bhmax }}=m_{p l} \frac{\tau}{t_{P l}} \delta_{0}^{-3 / 2}=m_{p l}^{2} \tau \delta_{0}^{-3 / 2}
$$

The probability, given by Equation (24), is also appropriate for the PBH formation in the matter dominated preheating stage after inflation $[3,4,196]$.

The probability $W_{P B H}(M)$ determines the fraction of total density

$$
\beta(M)=\frac{\rho_{P B H}(M)}{\rho_{t o t}} \approx W_{P B H}(M)
$$

corresponding to PBHs with mass $M$ is small for $\delta(M) \ll 1$. This means that the bulk of particles do not collapse directly into $\mathrm{PBHs}$, but form nonrelativistic gravitationally bound systems. The evolution of these systems strongly depends on particle properties but it can substantially enhance probability of $\mathrm{PBH}$ formation at early matter dominated stages.

\subsubsection{Evolutionary Formation of PBHs}

Gravitationally bound systems of collisionless gas resemble modern galaxies with collisionless gas of stars. Such a system is unstable relative to collapse to a black hole, but the corresponding evolution is a very slow process, being determined by the rate of dissipation due to evaporation of particles, whose velocity exceeds the excape velocity $[3,4,197]$. In the case of gravitational binary collisions, the evolution timescale $[3,4,197]$ is given by

$$
t_{e v}=\frac{N}{\ln N} t_{f f}
$$

for a gravitationally bound system of $N$ massive particles. Here $t_{f f}$ is the free fall time for a system with density $\rho$, which is given by $t_{f f} \approx(4 \pi G \rho)^{-1 / 2}$. due to Collective effects in collisionless gas [198] can make the evolution timescale shorter, being at large $N$ on the order of

$$
t_{e v} \sim N^{2 / 3} \cdot t_{f f}
$$

The free fall time for gravitationally bound systems of collisionless gas is on the order of cosmological time $t_{f}$ for the period when these systems are formed at the matter dominated stage. Therefore, even with the account for collective effects, the particles should be very long-living $\left(\tau \ll t_{f}\right)$ to form black holes in the evolutionary process.

Superheavy particles interacting with light relativistic particles and radiation form gravitationally bound systems with much smaller evolutionary time scale, than collisionless 
gas. Such systems have analogy with stars, in which evolution is determined by radiative energy loss. Such star-like systems could be formed at matter dominance of superheavy color octet fermions of asymptotically free SU(5) model [199] or magnetic monopoles of GUT models. In charge symmetric case, frozen out particles and antiparticles can annihilate in gravitationally bound systems. However, detailed numerical simulation [200] has shown that such annihilation cannot prevent the collapse of the majority of mass within these systems. The timescale of PBH formation by star-like objects does not exceed the cosmological time of the period when the systems are formed. The peak of these PBH spectrum corresponds to the mass within the cosmological horizon in the beginning of matter dominance, given by Equation (26).

\subsection{PBHs from Phase Transitions in the Inflationary Stage}

The data of the precision cosmology seem to favor red spectrum of density fluctuations with the amplitude of fluctuations decreasing to small scales. It confirms the prediction of single filed inflation. However this trend is based on the large-scale observations of Cosmic Microwave Background (CMB) and Large Scale Structure (LSS). On the other side, the observed strong inhomogeneity on scales, smaller than clusters of galaxies can easily mask strong deviations from this trend, reflecting the rich symmetry breaking pattern of BSM models. The presence of other scalar fields in the period of inflation can lead to spikes in the spectrum of density fluctuations on the scales determined by the parameters of the considered fields and their interaction with inflaton. This possibility was first pointed out in the chaotic inflation scenario in Reference [201]. It can strongly enhance probability of $\mathrm{PBH}$ formation on these scales, making PBH spectrum a sensitive probe for the existence of such fields.

This sensitivity can be illustrated by the example of phase transition induced by interaction of a Higgs field $\phi$ with an inflaton field $\eta$ (see Reference [31] for review and references). If this interaction induces positive mass term $+\frac{v^{2}}{2} \eta^{2} \phi^{2}$, at a certain critical value $\eta_{c}=m / v$ of the amplitude of the inflaton field the mass term in Higgs potential

$$
V(\phi, \eta)=-\frac{m_{\phi}^{2}}{2} \phi^{2}+\frac{\lambda_{\phi}}{4} \phi^{4}+\frac{v^{2}}{2} \eta^{2} \phi^{2}
$$

changes sign. At this stage of inflation, slow rolling phase transition takes place, which leads to the appearance of a characteristic spike in the spectrum of density fluctuations. These spike-like features generated at $e$-fold $(60 \geq N \geq 1)$, strongly enhance PBH formation at the corresponding scale. For the vacuum expectation value of a Higgs field

$$
\langle\phi\rangle=\frac{m}{\lambda}=v
$$

and $\lambda \sim 10^{-3}$, the amplitude $\delta$ of a spike is given by [202]

$$
\delta \approx \frac{4}{9 s}
$$

with

$$
s=\sqrt{\frac{4}{9}+\kappa 10^{5}\left(\frac{v}{m_{p l}}\right)^{2}}-\frac{3}{2}
$$

where $\kappa \sim 1$.

If the spike re-enters the horizon at the radiation dominated (RD) stage, it leads to enhancement of formation of PBHs with the mass

$$
M \approx \frac{m_{P l}^{2}}{H_{0}} \exp \{2 N\},
$$

where $H_{0}$ is the Hubble constant in the period of inflation. 
The spikes, re-entering horizon in the matter dominated (MD) stage, induce formation of PBHs of mass

$$
M \approx \frac{m_{P l}^{2}}{H_{0}} \exp \{3 N\}
$$

The model of horizontal unification [203-206] predicts succession of phase transitions of family symmetry breaking. At the high-energy scale of this breaking, such phase transitions should take place in the inflationary stage and enhancement of $\mathrm{PBH}$ formation by the corresponding spikes provides severe constraints on the scale of family symmetry breaking from the upper limits on PBHs of the corresponding mass [202].

\subsection{PBHs Form First Order Phase Transitions}

First order phase transitions in the early Universe go through nucleation of bubbles of true vacuum and their subsequent expansion in the false vacuum [207], in which potential energy of the false vacuum is converted into the kinetic energy of bubble walls. The bubble expands until it collides with another one. In a collision of several bubbles a black hole may be created [208,209]. Though the probability of the collision of two bubbles is much higher, strict conservation of the original $\mathrm{O}(2,1)$ symmetry seemed to prevent $\mathrm{PBH}$ formation in such processes. However, the investigations [210-212] revealed the mechanisms of breaking of this symmetry, making possible to create PBHs with a probability of order unity in collisions of only two bubbles. It makes $\mathrm{PBH}$ formation a sensitive probe for first-order phase transition in the early Universe.

If inflation models ended by a first-order phase transition (see for example [213-220]), the phase transition is completed after the true vacuum percolation regime is established, when at least one bubble per unit Hubble volume is nucleated, corresponding to the condition [220]:

$$
Q \equiv \frac{4 \pi}{9}\left(\frac{\Gamma}{H^{4}}\right)_{t_{\text {end }}}=1,
$$

where $\Gamma$ is the bubble nucleation rate and $H$ is the Hubble constant in the period of transition. If the two bubble collision leads to $\mathrm{PBH}$ formation, the mass of this $\mathrm{PBH}$ is given by [210-212]

$$
M_{B H}=\gamma_{1} M_{b u b}
$$

where $\gamma_{1} \simeq 10^{-2}$. Here $M_{b u b}$ is the mass within the bubble volume at the epoch of collision. Then collision between bubbles of Hubble size in the percolation regime would lead to copious PBH formation with masses

$$
M_{0}=\gamma_{1} M_{e n d}^{h o r}=\frac{\gamma_{1}}{2} \frac{m_{p l}^{2}}{H_{\text {end }}}
$$

where $M_{\text {end }}^{\text {hor }}$ is the mass within the Hubble horizon at the end of inflation, coinciding with $M_{b u b}$ in (39). Then the initial mass fraction of this PBHs is given by [210-212] $\beta_{0} \approx$ $\gamma_{1} / e \approx 6 \times 10^{-3}$. For example, for a typical value of $H_{\text {end }} \approx 4 \times 10^{-6} m_{p l}$, the initial mass fraction $\beta$ is contained in PBHs with mass $M_{0} \approx 1 \mathrm{~g}$. Such mini PBHs experience Hawking evaporation [221] at $t \sim 10^{-27} \mathrm{~s}$, but products of their evaporation can lead to observable effects.

If PBH vanishes completely [222], stable particles- products of evaporation can provide the test for their existence, as discussed in the next subsection. If $\mathrm{PBH}$ evaporation leaves a stable relic (see References [223-226]) with the mass of order $m_{r e l}=k m_{p l}\left(1 \leq k \leq 10^{2}\right)$ the constraints may be even more stronger [210-212] and with the account for the observational constraints on PBHs [227-233] can exclude the coexistence of stable remnants of $\mathrm{PBH}$ evaporation with the first-order phase transitions at the end of inflation.

The physics of dark Universe can lead to first-order phase transitions in the dark sectors. PBH formation in these transitions together with gravitational wave background can be an important cosmological probe for such transitions and the underlying symmetry 
breaking pattern of the dark sector. It makes necessary detailed analysis of mechanism of PBH formation in bubble collisions. In particular, the formation of PBH in bubble wall collisions was not found in lattice calculations [234], but these calculations did not take into account the evolution of false vacuum bag, studied in [235]. On the other hand, studies of this evolution found rather probable formation of oscillons, which do not collapse in PBHs. The open question of relationship between formation of oscillons and PBHs in association of gravitational wave background deserves special study.

\subsection{PBH Evaporation as Universal Particle Accelerator}

The possibility of $\mathrm{PBH}$ evaporation [221], strongly increases the sensitivity of the astrophysical data the existence of PBHs and mechanisms of their formation $[3,31,191,193,236]$. Constraining the PBH abundance provide unique information on the power spectrum on very small scales. PBHs with initial masses between $\sim 10^{9} \mathrm{~g}$ and $\sim 10^{16} \mathrm{~g}$ have led to stringent upper limits (see References $[31,191,223,237,238]$ ). These observational constraints follow either from the direct effects of the evaporated particles (for masses between $10^{14} \mathrm{~g}$ and $10^{16} \mathrm{~g}$ ) or from indirect effects of their interaction with matter and radiation (for PBH masses between $10^{9} \mathrm{~g}$ and $10^{14} \mathrm{~g}$ ). These effects can influence the entropy per baryon, the deuterium destruction, the ${ }^{4} \mathrm{He}$ destruction, and put contributions to the cosmic-rays by particles. However, since evaporation is originated by gravitational field, any particle, which can exist in our space-time and thus possess gravitational interaction can be emitted, provided that its mass does not exceed substantially the black hole temperature. Being independent of the strength of other particle interactions, $\mathrm{PBH}$ evaporation can provide copious production of super-weakly interacting particles. Such particles cannot be in equilibrium with the hot plasma and their primordial abundance cannot be frozen out. The only source of such particles in the early universe is their freeze, caused by the rare processes of their production by their super-weak interaction. Mini-PBH evaporation strongly enhances the frozen-in abundance of such particles.

The production of super-weakly interacting gravitino in the $\mathrm{PBH}$ evaporation $[3,4,236,239]$ (see also $[96,97]$ ) provides stringent constraints on PBHs with the mass $M \leq 10^{9} \mathrm{~g}$ and the corresponding mechanisms of their formation [98]. Such PBHs evaporate before BBN and can only influence entropy production, while production of gravitino in the PBH evaporation makes possible to use the astrophysical constraints on gravitino to probe the mechanisms of mini-PBH formation and BSM physics, underlying them.

\section{Discussion}

The basic elements of the modern cosmology find their physical nature in the dark sector of the fundamental physics. The corresponding cosmological impact reflects the underlying symmetry and symmetry breaking pattern in this sector.

If dark matter exists as the dominant form of nonrelativistic matter in the modern Universe, its stability should find some fundamental physical reason. We have given several examples of practical necessity of extension of the symmetry of the Standard model of elementary particles, which inevitably leads to predictions of new stable particles, playing the role of dark matter candidates. Taking into account the variety of motivations for such SM extensions, one can expect that dark matter can be multicomponent, involving all these particle candidates in the consideration. Then the question on the nature of dark matter transforms into the question on the form of dark matter, which plays the dominant dynamical role in formation and evolution of the cosmological Large Scale Structure.

Moreover, the necessity to solve the problem of strong $\mathrm{CP}$ violation in $\mathrm{QCD}$ gave rise to the prediction of QCD axion as dark matter candidate. It should behave as cold dark matter in spite of it's small mass, since axions would be created in the early Universe not as a primordial thermal gas, but as nonrelativistic Bose condensate of axions in the ground state. Creation of axions can possess large-scale correlations in its energy distribution, which leads to primordial nonlinear structures [29]. Phase transitions, predicted by the axion physics can be related with mechanisms of Primordial Black Hole (PBH) formation [31], 
including mechanisms of massive and even supermassive PBH formation $[31,160,161,167]$. PBHs in some mass range can be dominant dark matter candidate [240], but even if their contribution into the total density is subdominant, they can be together with primordial gravitational wave background important cosmological messenger of the physical nature of the dark matter.

A possibility to explain some effects of dark matter by modified gravity [241,242] extends the analysis beyond General relativity, the standard model of gravity, in which the method of multimessenger probes is also applicable.

To conclude, the solutions of the problem of the physical nature of the Dark Universe inevitably lead beyond the standard physical and cosmological models to their model-dependent multimessenger test in combination of their physical, astrophysical and cosmological signatures. The mystical Ouroboros (self-eating-snake) illustrated the main problem of modern fundamental physics: The theory of the universe is based on the predictions of fundamental physics, which in turn need cosmology for their test. Indeed, our modern understanding of the structure and evolution of the universe involves phenomena of inflation, baryosynthesis, and dark matter and dark energy. Cosmoparticle physics $[3-5,26,243]$ offers the way out of this vicious circle. It studies the fundamental basis and mutual relationship between micro-and macro-worlds in the proper combination of physical, astrophysical, and cosmological signatures. The approach of cosmoparticle physics can shed light on the dark Universe, which according to the modern paradigm was governed by unknown physics in its beginning and remains dominantly dark at the present stage of cosmological evolution.

Funding: The research was financially supported by Southern Federal University, 2020 Project VnGr/2020-03-IF.

Acknowledgments: I express my gratitude to Sergey V. Ketov for the kind invitation to contribute to this Special issue and to all my co-authors of the original papers, on which the present review is based.

Conflicts of Interest: The author declares no conflict of interest.

\section{References}

1. Weinberg, S. Gravitation and Cosmology; John Wiley and Sons, Inc.: New York, NY, USA, 1972.

2. Zeldovich, Y.B.; Novikov, I.D. Structure and Evolution of the Universe; Nauka: Moscow, Russia, 1985.

3. Khlopov, M.Y. Cosmoparticle Physics; World Scientific: Singapore, 1999.

4. Khlopov, M.Y. Fundamentals of Cosmoparticle Physics; CISP-Springer: Cambridge, UK, 2012.

5. Khlopov, M.Y. Cosmoparticle physics: Cross-disciplinary study of physics beyond the standard model. Bled Work. Phys. 2006, 7, 51-62.

6. Khlopov, M.Y. Direct and indirect astrophysical effects of hypothetical particles and fields. In Cosmion-94; Editions Frontieres: Moscow, Russia, 1996.

7. Khlopov, M.Y.; Mankoc-Borstnik, N.S. Proceedings to the 10th workshop 'what comes beyond the standard models. Bled Work. Phys. 2007, 8, 114-131.

8. Bertone, G. Particle Dark Matter: Observations, Models and Searches; Cambridge University Press: Cambridge, UK, 2010.

9. Frenk, C.S.; White, S.D.M. Dark matter and cosmic structure. Ann. Phys. 2012, 524, 507-534. [CrossRef]

10. Gelmini, G.B. Search for dark matter. Int. J. Mod. Phys. A 2008, 23, 4273-4288. [CrossRef]

11. Aprile, E.; Profumo, S. Focus on dark matter and particle physics. New J. Phys. 2009, 11, 105002. [CrossRef]

12. Feng, J.L. Dark matter candidates from particle physics and methods of detection. Ann. Rev. Astron. Astrophys. 2010, 48, 495-545. [CrossRef]

13. Starobinsky, A. A new type of isotropic cosmological models without singularity. Phys. Lett. B 1980, 91, 99-102. [CrossRef]

14. Guth, A.H. The inflationary universe: A possible solution to the horizon and flatness problems. Phys. Rev. D 1981, 23, 347-356. [CrossRef]

15. Linde, A.D. A new inflationary universe scenario: A possible solution of the horizon, flatness, homogeneity, isotropy and primordial monopole problems. Phys. Lett. B 1982, 108, 389-393. [CrossRef]

16. Albrecht, A.; Steinhardt, P.J. Cosmology for grand unified theories with radiatively induced symmetry breaking. Phys. Rev. Lett. 1982, 48, 1220-1223. [CrossRef]

17. Linde, A.D. Chaotic inflation. Phys. Lett. B 1983, 129, 177-181. [CrossRef]

18. Sakharov, A.D. Violation of CP invariance, c asymmetry, and baryon asymmetry of the universe. JETP Lett. 1967, 5, $24-27$. 
19. Kuzmin, V.A. CP-noninvariance and baryon asymmetry of the universe. JETP Lett. 1970, 12, 228-230.

20. Linde, A.D. Particle Physics and Inflationary Cosmology; Harwood: Chur, Switzerland, 1990.

21. Kolb, E.W.; Turner, M.S. The Early Universe; Addison-Wesley: Boston, MA, USA, 1990.

22. Gorbunov, D.S.; Rubakov, V.A. Introduction to the Theory of the Early Universe Hot Big Bang Theory; World Scientific: Singapore, 2011.

23. Gorbunov, D.S.; Rubakov, V.A. Introduction to the Theory of the Early Universe. Cosmological Perturbations and Inflationary Theory; World Scientific: Singapore, 2011.

24. Carrasco, J.J.M.; Kallosh, R.; Linde, A. Minimal supergravity inflation. Phys. Rev. D 2016, 93, 061301. [CrossRef]

25. Khlopov, M.Y. Fundamental particle structure in the cosmological dark matter. Int. J. Mod. Phys. A 2013, 28, 1330042. [CrossRef]

26. Khlopov, M.Y. Fundamental cross-disciplinary studies of microworld and Universe. Vestn. Russ. Acad. Sci. 2001, $71,1133-1137$.

27. Khlopov, M. Cosmological Reflection of Particle Symmetry. Symmetry 2016, 8, 81. [CrossRef]

28. Khlopov, M. Multi-messenger cosmology of new physics. J. Phys. Conf. Ser. 2020, 1690, 012182. [CrossRef]

29. Khlopov, M. What comes after the Standard model? Prog. Part. Nucl. Phys. 2021, 116, 103824. [CrossRef]

30. Ketov, S.V.; Khlopov, M.Y. Cosmological Probes of Supersymmetric Field Theory Models at Superhigh Energy Scales. Symmetry 2019, 11, 511. [CrossRef]

31. Khlopov, M.Y. Primordial black holes. Res. Astron. Astrophys. 2010, 10, 495-528. [CrossRef]

32. Khlopov, M.Y. Cosmological probes for supersymmetry. Symmetry 2015, 7, 815-842. [CrossRef]

33. Yanagida, T. Horizontal Symmetry and Masses of Neutrinos. Prog. Theor. Phys. 1980, 64, 1103-1105. [CrossRef]

34. Schechter, J.; Valle, J.W.F. Neutrino masses in SU(2)x U(1) theories. Phys. Rev. D 1980, 22, 2227. [CrossRef]

35. Mohapatra, R.N.; Senjanović, G. Neutrino Mass and Spontaneous Parity Nonconservation. Phys. Rev. Lett. 1980, $44,912$. [CrossRef]

36. Zeldovich, Y.B.; Khlopov, M.Y. The neutrino mass in elementary particle physics and in big Bang cosmology. Sov. Phys. Uspekhi 1981, 24, 755-774. [CrossRef]

37. Merle, A. keV neutrino model building. Int. J. Mod. Phys. D 2013, 22, 1330020. [CrossRef]

38. Dasgupta, B.; Kopp, J. Sterile neutrinos. Phys. Rep. 2021, 928, 1-63. [CrossRef]

39. Aguilar-Arevalo, A.; Auerbach, L.B.; Burman, R.L.; Caldwell, D.O.; Church, E.D.; Cochran, A.K.; Donahue, J.B.; Fazely, A.; Garvey, G.T.; Gunasingha, R.M.; et al. Evidence for neutrino oscillations from the observation of $v_{e}$ appearance in a $v_{\mu}$ beam. Phys. Rev. D 2001, 64, 112007. [CrossRef]

40. Aguilar-Arevalo, A.A.; Brown, B.C.; Conrad, J.M.; Dharmapalan, R.; Diaz, A.; Djurcic, Z.; Finley, D.A.; Ford, R.; Garvey, G.T.; Gollapinni, S.; et al. Updated MiniBooNE neutrino oscillation results with increased data and new background studies. Phys. Rev. D 2021, 103, 052002. [CrossRef]

41. Abratenko, P.; An, R.; Anthony, J.; Arellano, L.; Asaadi, J.; Ashkenazi, A.; Balasubramanian, S.; Baller, B.; Barnes, C.; Barr, G.; et al. Search for Neutrino-Induced Neutral Current Radiative Decay in MicroBooNE and a First Test of the MiniBooNE Low Energy Excess Under a Single-Photon Hypothesis. arXiv 2021, arXiv:2110.00409.

42. Argüelles, C.A.; Esteban, I.; Hostert, M.; Kelly, K.J.; Kopp, J.; Machado, P.A.N.; Martinez-Soler, I.; Perez-Gonzalez, Y.F. MicroBooNE and the $v_{e}$ Interpretation of the MiniBooNE Low-Energy Excess. arXiv 2021, arXiv:2111.10359

43. Kuzmin, V.A.; Rubakov, V.A.; Shaposhnikov, M.E. On anomalous electroweak baryon-number non-conservation in the early universe. Phys. Lett. B 1985, 155, 36-42. [CrossRef]

44. Canetti, L.; Drewes, M.; Shaposhnikov, M. Sterile Neutrinos as the Origin of Dark and Baryonic Matter. Phys. Rev. Lett. 2013, 110, 061801. [CrossRef]

45. Boyarsky, A.; Drewes, M.; Lasserre, T.; Mertens, S.; Ruchayskiy, O. Sterile neutrino Dark Matter. Prog. Part. Nucl. Phys. 2019, 104, 1-45. [CrossRef]

46. Bulbul, E.; Markevitch, M.; Foster, A.; Smith, R.K.; Loewenstein, M.; Randall, S.W. Detection of An Unidentified Emission Line in the Stacked X-ray spectrum of Galaxy Clusters. Astrophys. J. 2014, 789, 13. [CrossRef]

47. Ruchayskiy, O.; Boyarsky, A.; Iakubovskyi, D.; Bulbul, E.; Eckert, D.; Franse, J.; Maly-shev, D.; Markevitch, M.; Neronov, A. Searching for decaying dark matter in deep XMM-Newton observation of the Draco dwarf spheroidal. Mon. Not. R. Astron. Soc. 2016, 460, 1390-1398. [CrossRef]

48. Lee, T.D.; Yang, C.N. Question of parity conservation in weak interactions. Phys. Rev. 1956, 104, 254-258. [CrossRef]

49. Kobzarev, I.Y.; Okun, L.B.; Pomeranchuk, I.Y. On the possibility of experimental observation of mirror particles. Sov. J. Nucl. Phys. 1966, 3, 837-841.

50. Foot, R.; Volkas, R.R. Neutrino physics and the mirror world: How exact parity symmetry explains the solar neutrino deficit, the atmospheric neutrino anomaly and the LSND experiment. Phys. Rev. D 1995, 52, 6595-6606. [CrossRef]

51. Blinnikov, S.I.; Khlopov, M.Y. On possible effects of 'mirror' particles. Sov. J. Nucl. Phys. 1982, 36, $472-474$.

52. Blinnikov, S.I.; Khlopov, M.Y. Possible astronomical effects of mirror particles. Sov. Astron. J. 1983, 27, 371-375.

53. Berezhiani, Z.; Lepidi, A. Cosmological bounds on the 'millicharges' of mirror particles. Phys. Lett. B 2009, 681, 276. [CrossRef]

54. Carlson, E.D.; Glashow, S.L. Nucleosynthesis versus the mirror universe. Phys. Lett. B 1987, 193, 168-170. [CrossRef]

55. Foot, R.; Volkas, R.R. The Exact parity symmetric model and big bang nucleosynthesis. Astropart. Phys. 1997, 7, $283-295$. [CrossRef]

56. Berezhiani, Z.; Comelli, D.; Villante, F. The Early mirror universe: Inflation, baryogenesis, nucleosynthesis and dark matter. Phys. Lett. B 2001, 503, 362-375. [CrossRef] 
57. Berezhiani, Z. Mirror world and its cosmological consequences. Int. J. Mod. Phys. A 2004, 19, 3775-3806. [CrossRef]

58. Okun, L.B. Mirror particles and mirror matter: 50 years of speculations and search. Phys. Usp. 2007, 50, 380-389. [CrossRef]

59. Kolb, E.W.; Seckel, D.; Turner, M.S. The shadow world. Nature 1985, 314, 415. [CrossRef]

60. Khlopov, M.Y.; Beskin, G.M.; Bochkarev, N.G.; Pustilnik, L.A.; Pustilnik, S.A. Observational physics of mirror world. Sov. Astron. 1991, 35, 21-30.

61. Gervais, J.-L.; Sakita, B. Field Theory Interpretation of Supergauges in Dual Models. Nucl. Phys. B 1971, 34, 632-639. [CrossRef]

62. Golfand, Y.A.; Likhtman, E.P. Extension of the Algebra of Poincare Group Generators and Violation of P Invariance. JETP Lett. 1971, 13, 323-326.

63. Volkov, D.V.; Akulov, V.P. Possible universal neutrino interaction. JETP Lett. 1972, 16, 438-440.

64. Volkov, D.V.; Akulov, V.P. Is the Neutrino a Goldstone Particle? Phys. Lett. B 1973, 46, 109-110. [CrossRef]

65. Wess, J.; Zumino, B. A lagrangian model invariant under supergauge transformations. Phys. Lett. B 1974, 49, 52-54. [CrossRef]

66. Wess, J.; Zumino, B. Supergauge Transformations in Four-Dimensions. Nucl. Phys. B 1974, 70, 39-50. [CrossRef]

67. Baer, H.; Barger, V.; Gainer, J.S.; Sengupta, D.H.; Tata, X. LHC luminosity and energy upgrades confront natural supersymmetry models. Phys. Rev. D 2018, 98, 075010 [CrossRef]

68. Bernabei, R.; Bellia, P.; d'Angelo, S.; Di Marco, A.; Montecchia, F.; Cappella, F.; d'Angelo, A.; Incicchitti, A.; Caracciolo, V.; Castellano, S. Dark Matter investigation by DAMA at Gran Sasso. Int. J. Mod. Phys. A 2013, 28, 1330022. [CrossRef]

69. Abrams, D.; Akerib, D.S.; Barnes, P.D., Jr. Exclusion limits on the WIMP nucleon cross-section from the cryogenic dark matter search. Phys. Rev. D 2002, 66, 122003. [CrossRef]

70. Akerib, D.S.; Armel-Funkhouser, M.S.; Attisha, M.J.; Young, B.A. Exclusion limits on the WIMP-nucleon cross section from the first run of the cryogenic dark matter search in the soudan underground laboratory. Phys. Rev. D 2005, 72, 052009. [CrossRef]

71. Ahmed, Z.; Akerib, D.S.; Arrenberg, S. Search for weakly interacting massive particles with the first five-tower data from the cryogenic dark matter search at the soudan underground laboratory. Phys. Rev. Lett. 2009, 102, 011301. [CrossRef]

72. Aprile, E.; Arisaka, K.; Arneodo, F. First dark matter results from the XENON100 experiment. Phys. Rev. Lett. 2010, 105, 131302. [CrossRef] [PubMed]

73. Aalseth, C.E.; Barbeau, P.S.; Bowden, N.S. Results from a search for light-mass dark matter with a p-type point contact germanium detector. Phys. Rev. Lett. 2011, 106, 131302. [CrossRef] [PubMed]

74. Angloher, G.; Bauer, M.; Bavykina, I.; Bento, A.; Bucci, C.; Ciemniak, C.; Deuter, G.; von Feilitzsch, F.; Hauff, D.; Huffet, P. Results from $730 \mathrm{~kg}$ days of the CRESST-II Dark Matter search. Eur. Phys. J. C 2012, 72, 1971. [CrossRef]

75. Akerib, D.; Alsum, S.; Araujo, H.M.; Bai, X.; Balajthy, J.; Beltrame, P.; Bernard, E.P.; Bernstein, A.; Biesiadzinski, T.P. Search for annual and diurnal rate modulations in the LUX experiment. Phys. Rev. D 2018, 98, 062005. [CrossRef]

76. Cheng, C.; Xie, P.; Abdukerim, A.; Chen, W.; Chen, X.; Chen, Y.; Cui, X.; Fan, Y.; Fang, D.; Fu, C.; et al. Search for Light Dark Matter-Electron Scattering in the PandaX-II Experiment. Phys. Rev. Lett. 2021, 126, 211803. [CrossRef] [PubMed]

77. Adhikari, G.; Barbosa de Souza, E.; Carlin, N.; Choi, J.J.; Choi, S.; Djamal, M.; Ezeribe, A.C.; França, L.E.; Ha, C.; Hahn, I.S.; et al. Strong constraints from COSINE-100 on the DAMA dark matter results using the same sodium iodide target. Sci. Adv. 2021, 7, eabk2699. [CrossRef]

78. Zeldovich, Y.B.; Klypin, A.A.; Khlopov, M.Y.; Chechetkin, V.M. Astrophysical bounds on the mass of heavy stable neutral leptons. Sov. J. Nucl. Phys. 1980, 31, 664-669.

79. Jungman, G.; Kamionkowski, M.; Griest, K. Supersymmetric dark matter. Phys. Rep. 1996, 267, 195-373. [CrossRef]

80. Fargion, D.; Khlopov, M.Y.; Konoplich, R.V.; Mignani, R. Bounds on very heavy relic neutrinos by their annihilation in galactic halo. Phys. Rev. D 1995, 52, 1828-1836. [CrossRef]

81. Adriani, O.; Barbarino, G.C.; Bazilevskaya, G.A.; Bellotti, R.; Boezio, M.; Bogomolov, E.A.; Bonechi, L.; Bongi, M.; Bonvicini, V.; Bottai, S. An anomalous positron abundance in cosmic rays with energies 1.5-100 GeV. Nature 2009, 458, 607-609. [CrossRef]

82. Aguilar, M.; Alberti, G.; Alpat, B. First result from the alpha magnetic spectrometer on the international space station: Precision measurement of the positron fraction in primary cosmic rays of 0.5-350 GeV. Phys. Rev. Lett. 2013, 110, 141102. [CrossRef] [PubMed]

83. Aguilar, M.; Aisa, D.; Alvino, A.; Ambrosi, G.; Andeen, K.; Arruda, L.; Attig, N.; Azzarello, P.; Bachlechner, A.; Barao F.; et al. Electron and Positron Fluxes in Primary Cosmic Rays Measured with the Alpha Magnetic Spectrometer on the International Space Station. Phys. Rev. Lett. 2014, 113, 121102. [CrossRef]

84. Accardo, L.; Aguilar, M.; Aisa, D.; Alpat, B.; Alvino, A.; Ambrosi, G.; Andeen, K.; Arruda, L.; Attig, N.; Azzarello, P.; et al. High Statistics Measurement of the Positron Fraction in Primary Cosmic Rays of 0.5-500 GeV with the Alpha Magnetic Spectrometer on the International Space Station. Phys. Rev. Lett. 2014, 113, 121101. [CrossRef]

85. Khlopov, M. Direct and Indirect Probes for Composite Dark Matter. Front. Phys. 2019, 7, 4. [CrossRef]

86. Ackermann, M.; Ajello, M.; Albert, A.; Baldini, L.; Barbiellini, G.; Bechtol, K.; Bellazzini, R.; Berenji, B.; Blandford, R.D.; Bloom, E.D.; et al. Fermi LAT Search for Dark Matter in Gamma-ray Lines and the Inclusive Photon Spectrum. Phys. Rev. D 2012, 86, 022002. [CrossRef]

87. Belotsky, K.M.; Kamaletdinov, A.K.; Shlepkina, E.S.; Solovyov, M.L. Cosmic Gamma Ray Constraints on the Indirect Effects of Dark Matter. Particles 2020, 3, 336-344. [CrossRef]

88. Freese, K. Can scalar neutrinos or massive Dirac neutrinos be the missing mass? Phys. Lett. B 1986, 167, 295-300. [CrossRef]

89. Khlopov, M.Y.; Linde, A.D. Is it easy to save gravitino? Phys. Lett. B 1984, 138, 265-268. [CrossRef] 
90. Balestra, F. Annihilation of antiprotons with Helium-4 at low energies and its relationship with the problems of the modern cosmology and models of grand unification. Sov. J. Nucl. Phys. 1984, 39, 626-631.

91. Levitan, Y.L.; Sobol, I.M.; Khlopov, M.Y.; Chechetkin, V.M. Production of light elements in cascades from energetic antiprotons in early Universe and problems of nuclear cosmoarcheology. Sov. J. Nucl. Phys. 1988, 47, 109-115.

92. Khlopov, M.Y.; Levitan, Y.L.; Sedel'Nikov, E.V.; Sobol, I.M. Nonequilibrium cosmological nucleosynthesis of light elements: Calculations by the Monte Carlo method. Phys. Atom. Nucl. 1994, 57, 1393-1397.

93. Sedel'nikov, E.V.; Filippov, S.S.; Khlopov, M.Y. Kinetic theory of nonequilibrium cosmological nucleosynthesis. Phys. Atom. Nucl. $1995,58,235-242$.

94. Jedamzik, K. Did something decay, evaporate, or annihilate during Big Bang nucleosynthesis? Phys. Rev. D 2004, 70, 063524. [CrossRef]

95. Kawasaki, M.; Kohri, K.; Moroi, T. Hadronic decay of late-decaying particles and Big-Bang nucleosynthesis. Phys. Lett. 2005, B625, 7-12. [CrossRef]

96. Lemoine, M. Moduli constraints on primordial black holes. Phys. Lett. B 2000, 481, 333-338. [CrossRef]

97. Green, A.M. Supersymmetry and primordial black hole abundance constraints. Phys. Rev. D 1998, 60, 063516. [CrossRef]

98. Khlopov, M.Y.; Barrau, A.; Grain, J. Gravitino production by primordial black hole evaporation and constraints on the inhomogeneity of the early Universe. Class. Quantum Grav. 2006, 23, 1875-1882. [CrossRef]

99. Addazi, A.; Marciano, A.; Ketov, S.V.; Khlopov, M.Y. Physics of superheavy dark matter in supergravity. Int. J. Mod. Phys. D 2018, 27, 1841011. [CrossRef]

100. Kaplan, D.B.; Georgi, H. SU(2) × U(1) Breaking by Vacuum Misalignment. Phys. Lett. B 1984, 136, 183-186. [CrossRef]

101. Kaplan, D.B.; Georgi, H.; Dimopoulos, S. Composite Higgs Scalars. Phys. Lett. B 1984, 136, 187-190. [CrossRef]

102. Arkani-Hamed, N.; Cohen, A.G.; Katz, E.; Nelson, A.E.; Gregoire, T.; Wacker, J.G. The minimal moose for a little Higgs. J. High Energy Phys. 2002, 8, 021. [CrossRef]

103. Arkani-Hamed, N.; Cohen, A.G.; Katz, E.; Nelson, A.E. The Littlest Higgs. J. High Energy Phys. 2002, 7, 034. [CrossRef]

104. Agashe, K.; Contino, R.; Pomarol, A. The minimal composite Higgs model. Nucl. Phys. B 2005, 719, 165-187. [CrossRef]

105. Contino, R.; Da Rold, L.; Pomarol, A. Light custodians in natural composite Higgs models. Phys. Rev. D 2007, 75, 055014. [CrossRef]

106. Kats, Y.; McCullough, M.; Perez, G.; Soreq, Y.; Thaler, J. Colorful Twisted Top Partners and Partnerium at the LHC. J. High Energy Phys. 2017, 6, 126. [CrossRef]

107. Jaeger, S.; Kvedaraite, S.; Perez, G.; Savoray, I. Bounds and prospects for stable multiply charged particles at the LHC. J. High Energy Phys. 2019, 2019, 41. [CrossRef]

108. Fargion, D.; Khlopov, M.Y.; Stephan, C.A. Cold dark matter by heavy double charged leptons? Class. Quantum Grav. 2006, 23, 7305-7354.

109. Connes, A. Noncommutative Geometry; Academic Press: San Diego, CA, USA, 1994.

110. Khlopov, M.Y.; Kouvaris, C. Strong interactive massive particles from a strong coupled theory. Phys. Rev. D 2008, 77, 065002. [CrossRef]

111. Sannino, F.; Tuominen, K. Orientifold theory dynamics and symmetry breaking. Phys. Rev. D 2005, 71, 051901. [CrossRef]

112. Hong, D.K.; Hsu, S.D.H.; Sannino, F. Composite higgs from higher representations. Phys. Lett. B 2004, 597, 89-93. [CrossRef]

113. Dietrich, D.D.; Sannino, F.; Tuominen, K. Light composite higgs from higher representations versus electroweak precision measurements: Predictions for LHC. Phys. Rev. D 2005, 72, 055001. [CrossRef]

114. Dietrich, D.D.; Sannino, F.; Tuominen, K. Light composite higgs and precision electroweak measurements on the Z resonance: An update. Phys. Rev. D 2006, 73, 037701. [CrossRef]

115. Gudnason, S.B.; Kouvaris, C.; Sannino, F. Towards working technicolor: Effective theories and dark matter. Phys. Rev. D 2006, 73, 115003. [CrossRef]

116. Gudnason, S.B.; Kouvaris, C.; Sannino, F. Dark matter from new technicolor theories. Phys. Rev. D 2006, 74, 095008. [CrossRef]

117. Khlopov, M.Y. Composite dark matter from 4th generation. JETP Lett. 2006, 83, 1-4. [CrossRef]

118. Mankoč Borštnik, N.S. The spin-charge-family theory is explaining the origin of families, of the Higgs and the Yukawa couplings. Bled Works. Phys. 2010, 11, 105-129.

119. Goertz, F. Composite Higgs theory. PoS 2018, ALPS2018, 12. [CrossRef]

120. Khlopov, M.Y.; Shibaev, R.M. Probes for 4th generation constituents of dark atoms in Higgs boson studies at the LHC. Adv. High Energy Phys. 2014, 2014, 406458. [CrossRef]

121. Khlopov, M.Y.; Kouvaris, C. Composite dark matter from a model with composite Higgs boson. Phys. Rev. D 2008, 78, 065040. [CrossRef]

122. Kohri, K.; Takayama, F. Big bang nucleosynthesis with long-lived charged massive particles. Phys. Rev. D 2007, 76, 063507. [CrossRef]

123. Cahn R.N.; Glashow, S.L. Chemical signatures for superheavy elementary particles. Science 1981, 213, 607-611. [CrossRef]

124. Pospelov, M. Particle physics catalysis of thermal big bang nucleosynthesis. Phys. Rev. Lett. 2007, 98, 231301. [CrossRef]

125. Beylin, V.; Khlopov, M.; Kuksa, V.; Volchaanskiy, N. New physics of strong interaction and Dark Universe. Universe 2020, 6, 196. [CrossRef] 
126. Bikbaev, T.E.; Khlopov, M.Y.; Mayorov, A.G. Numerical simulation of dark atom interaction with nuclei. Bled Work. Phys. 2020, 21, 105-117.

127. Cudell, J.-R.; Khlopov, M.Y.; Wallemacq, Q. The nuclear physics of OHe. Bled Work. Phys. 2012, 13, 10-27.

128. Cudell, J.-R.; Khlopov, M.Y.; Wallemacq, Q. Some Potential Problems of OHe Composite Dark Matter. Bled Work. Phys. 2014, 15, 66-74.

129. Gani, V.A.; Khlopov, M.Y.; Voskresensky, D.N. Double charged heavy constituents of dark atoms and superheavy nuclear objects. Phys. Rev. D 2019, 99, 015024. [CrossRef]

130. De Lavallaz, A.; Fairbairn, M. Neutron stars as dark matter probes. Phys. Rev. D 2010, 81, 123521. [CrossRef]

131. Cudell, J.-R.; Khlopov, M.Y.; Wallemacq, Q. Dark atoms and the positron-annihilation-line excess in the galactic bulge. Adv. High Energy Phys. 2014, 2014, 869425. [CrossRef]

132. Finkbeiner, D.P.; Weiner, N. Exciting Dark Matter and the INTEGRAL/SPI 511 keV signal. Phys. Rev. D 2007, 76, 083519. [CrossRef]

133. Teegarden, B.J.; Watanabe, K.; Jean, P.; Knodlseder, J.; Lonjou, V.; Roques, J.P.; Skinner, G.K.; von Ballmoos, P.; Weidenspointner, G.; Bazzano, A.; et al. INTEGRAL/SPI Limits on Electron-Positron Annihilation Radiation from the Galactic Plane. Astrophys. J. 2005, 621, 296-300. [CrossRef]

134. Khlopov, M. Removing the conspiracy of BSM physics and BSM cosmology. Int. J. Mod. Phys. D 2019, 28, 1941012. [CrossRef]

135. Fairbairn, M.; Kraan, A.C.; Milstead, D.A.; Sjöstrand, T.; Skands, P.; Sloan, T. Stable Massive Particles at Colliders. Phys. Rep. 2007, 438, 1-63. [CrossRef]

136. ATLAS Collaboration. Search for heavy long-lived multi-charged particles in proton-proton collisions at $\sqrt{s}=13$ TeV using the ATLAS detector. Phys. Rev. D 2019, 99, 052003. [CrossRef]

137. T'Hooft, G. Magnetic monopoles in unified gauge theories. Nucl. Phys. B 1974, 79, $276-284$.

138. Polyakov, A.M. Particle spectrum in quantum field theory. JETP Lett. 1974, 20, 194-195.

139. Zeldovich, Y.B.; Khlopov, M.Y. On the concentration of relic magnetic monopoles in the universe. Phys. Lett. 1978, B79, $239-242$. [CrossRef]

140. Khlopov, M.Y. Primordial magnetic monopoles. Priroda 1979, 12, 99-101.

141. Preskill, J.P. Cosmological production of superheavy magnetic monopoles. Phys. Rev. Lett. 1979, 43, 1365-1374. [CrossRef]

142. Khlopov, M.Y. On the concentration of magnetic monopoles in the universe. In Magnetic Stars; Nauka: Leningrad, Russia, 1988; p. 201.

143. Zeldovich, Y.B. Cosmological fluctuations produced near a singularity. Mon. Not. R. Astr. Soc. 1980, 192, 663-667. [CrossRef]

144. Vilenkin, A. Cosmological density fluctuations produced by vacuum strings. Phys. Rev. Lett. 1981, 46, 1169-1172. [CrossRef]

145. Zeldovich, Y.B.; Kobzarev, I.Y.; Okun, L.B. Cosmological consequences of the spontaneous breakdown of discrete symmetry. Sov. Phys. JETP 1975, 40, 1-5.

146. Peccei, R.D.; Quinn, H.R. CP Conservation in the Presence of Pseudoparticles. Phys. Rev. Lett. 1977, 38, 1440-1443. [CrossRef]

147. Weinberg, S. A New Light Boson? Phys. Rev. Lett. 1978, 40, 223. [CrossRef]

148. Wilczek, F. Problem of Strong P and T Invariance in the Presence of Instantons. Phys. Rev. Lett. 1978, 40, 279. [CrossRef]

149. Marsh, D.J.E. Axion Cosmology. Phys. Rep. 2016, 643, 1-79. [CrossRef]

150. Di Luzio, L.; Giannotti, M.; Nardi, E.; Visinelli, L. The landscape of QCD axion models. Phys. Rep. 2020, 870, 1-117. [CrossRef]

151. Sakharov, A.S.; Khlopov, M.Y. The nonhomogeneity problem for the primordial axion field. Phys. Atom. Nucl. 1994, 57, 485-487.

152. Sakharov, A.S.; Khlopov, M.Y.; Sokoloff, D.D. Large scale modulation of the distribution of coherent oscillations of a primordial axion field in the Universe. Phys. Atom. Nucl. 1996, 59, 1005-1010.

153. Sakharov, A.S.; Khlopov, M.Y.; Sokoloff, D.D. The nonlinear modulation of the density distribution in standard axionic CDM and its cosmological impact. Nucl. Phys. B Proc. Suppl. 1999, 72, 105-109.

154. Jaeckel, J.; Ringwald, A. The low-energy frontier of particle physics. Ann. Rev. Nucl. Part. Sci. 2010, 60, 405-437. [CrossRef]

155. Kim, J.E. Light pseudoscalars, particle physics and cosmology. Phys. Rep. 1987, 150, 1-177. [CrossRef]

156. Vilenkin, A.; Shellard, E.P.S. Cosmic Strings and Other Topological Defects; Cambridge University Press: Cambridge, UK, 1994.

157. Rubin, S.G.; Khlopov, M.Y.; Sakharov, A.S. Primordial black holes from non-equilibrium second order phase transitions. Gravit. Cosmol. Suppl. 2000, 6, 51-58.

158. Khlopov, M.Y.; Rubin, S.G. Cosmological Pattern of Microphysics in Inflationary Universe; Kluwer: Dordrecht, The Netherlands, 2004.

159. Khlopov, M.Y.; Rubin, S.G.; Sakharov, A.S. Strong primordial nonhomogeneities and galaxy formation. Gravit. Cosmol. Suppl. 2002, 8, 57-65.

160. Belotsky, K.M.; Dokuchaev, V.I.; Eroshenko, Y.N.; Esipova, E.A.; Khlopov, M.Y.; Khromykh, L.A.; Kirillov, A.A.; Nikulin, V.V.; Rubin, S.G.; Svadkovsky, I.V. Clusters of primordial black holes. Eur. Phys. J. C 2019, 79, 246. [CrossRef]

161. Rubin, S.G.; Sakharov, A.S.; Khlopov, M.Y. Formation of primordial galactic nuclei at phase transitions in the early Universe. J. Exp. Theor. Phys. 2001, 92, 921-929. [CrossRef]

162. Dokuchaev, V.; Eroshenko, Y.; Rubin, S. Quasars formation around clusters of primordial black holes. Grav. Cosmol. 2005, 11, 99-104.

163. Khlopov, M.Y.; Rubin, S.G.; Sakharov, A.S. Primordial structure of massive black hole clusters. Astropart. Phys. 2005, 23, 265-277. [CrossRef] 
164. The LIGO Scientific Collaboration; The Virgo Collaboration; Abbott, B.P. GWTC-1: A gravitational-wave transient catalog of compact binary mergers observed by LIGO and Virgo during the first and second observing runs. arXiv 2018, arXiv:1811.12907.

165. Abbott, B.P.; Abbott, T.D.; Abraham, S.; Acernese, F.; Ackley, K.; Adams, A.; Adams, C.; Adhikari, R.X.; Adya, V.B.; Affeldt, C.; et al. GWTC-2: Compact Binary Coalescences Observed by LIGO and Virgo During the First Half of the Third Observing Run. arXiv 2020, arXiv:2010.14527.

166. Nitz, A.H.; Capano, C.D.; Kumar, S.; Wang, Y.-F.; Kastha, S.; Schäfer, M.; Dhurkunde, R.; Cabero, M. 3-OGC: Catalog of gravitational waves from compact-binary mergers. arXiv 2021, arXiv:2105.09151.

167. Dolgov, A.D. Massive primordial black holes in contemporary and young universe (old predictions and new data). Int. J. Mod. Phys. A 2018, 33, 1844029. [CrossRef]

168. Khlopov, M.Y. Multimessenger Probes for New Physics in Light of A. Sakharov's Legacy in Cosmoparticle Physics. Universe 2021, 7, 222. [CrossRef]

169. The LIGO Scientific Collaboration; The Virgo Collaboration; Abbott, R.; Abbott, T.D.; Abraham, S.; Acernese, F.; Ackley, K.; Adams, C.; Adhikari, R.X.; Adya, V.B.; et al. GW190521: A Binary Black Hole Merger with a Total Mass of 150 M $\odot$. Phys. Rev. Lett. 2020, 125, 101102. [CrossRef] [PubMed]

170. The LIGO Scientific Collaboration; The Virgo Collaboration; Abbott, R.; Abraham, S.; Acernese, F.; Ackley, K.; Adams, C.; Adhikari, R.X.; Adya, V.B.; Affeldt, C.; et al. Properties and astrophysical implications of the 150 Msun binary black hole merger GW190521. Astrophys. J. Lett. 2020, 900, L13. [CrossRef]

171. Bringmann, T.; Depta, P.F.; Domcke, V.; Schmidt-Hoberg, K. Strong constraints on clustered primordial black holes as dark matter. Phys. Rev. D 2019, 99, 063532. [CrossRef]

172. Chechetkin, V.M.; Khlopov, M.Y.; Sapozhnikov, M.G.; Zeldovich, Y.B. Astrophysical aspects of antiproton interaction with He (Antimatter in the Universe). Phys. Lett. B 1982, 118, 329-332. [CrossRef]

173. Dolgov, A.D. Matter and antimatter in the universe. Nucl. Phys. Proc. Suppl. 2002, 113, 40-49. [CrossRef]

174. Khlopov, M.Y.; Rubin, S.G.; Sakharov, A.S. Possible origin of antimatter regions in the baryon dominated universe. Phys. Rev. D 2000, 62, 083505. [CrossRef]

175. Dolgov, A.; Silk, J. Baryon isocurvature fluctuations at small scales and baryonic dark matter. Phys. Rev. D 1993, 47, 4244-4255. [CrossRef]

176. Dolgov, A.D.; Kawasaki, M.; Kevlishvili, N. Inhomogeneous baryogenesis, cosmic antimatter, and dark matter. Nucl. Phys. B 2009, 807, 229-250. [CrossRef]

177. Khlopov, M.Y. Primordial nonlinear structures and massive black holes from early Universe. J. Phys. Conf. Ser. $2007,66,012032$. [CrossRef]

178. Khlopov, M.Y. An antimatter globular cluster in our galaxy: A probe for the origin of matter. Gravit. Cosmol. 1998, 4, 69-72.

179. Golubkov, Y.A.; Khlopov, M.Y. Anti-protons annihilation in the galaxy as a source of diffuse gamma background. Phys. Atom. Nucl. 2001, 64, 1821-1829. [CrossRef]

180. Belotsky, K.M.; Golubkov, Y.A.; Khlopov, M.Y.; Konoplich, R.V.; Sakharov, A.S. Anti-helium flux as a signature for antimatter globular clusters in our galaxy. Phys. Atom. Nucl. 2000, 63, 233-239. [CrossRef]

181. Poulin, V.; Salati, P.; Cholis, I.; Kamionkowski, M.; Silk, J. Where do the AMS-02 anti-helium events come from? Phys. Rev. D 2019, 99, 023016. [CrossRef]

182. Fargion, D.; Khlopov, M.Y. Antimatter bounds by anti-asteroids annihilations on planets and sun. Astropart. Phys. 2003, 19, 441-446. [CrossRef]

183. Blinnikov, S.I.; Dolgov, A.D.; Postnov, K.A. Antimatter and antistars in the universe and in the Galaxy. Phys. Rev. D 2015, 92, 023516. [CrossRef]

184. Oppenheimer, J.R.; Sneider, H. On continued gravitational contraction. Phys. Rev. 1939, 56, 455-459. [CrossRef]

185. Zeldovich, Y.B.; Novikov, I.D. Relativistic Astrophysics, Volume 1: Stars and Relativity; University of Chicago Press: Chicago, IL, USA, 1971.

186. Zeldovich, Y.B.; Novikov, I.D. The hypothesis of cores retarded during expansion and the hot cosmological model. Sov. Astron. 1967, 10, 602-609.

187. Hawking, S.W. Gravitationally collapsed objects of very low mass. Mon. Not. R. Astron. Soc. 1971, 152, 75-78. [CrossRef]

188. Carr, B.J.; Hawking, S.W. Black holes in the early Universe. Mon. Not. R. Astron. Soc. 1974, 168, 399-415. [CrossRef]

189. Carr, B.J. The primordial black hole mass spectrum. Astroph. J. 1975, 201, 1-19. [CrossRef]

190. Khlopov, M.Y.; Polnarev, A.G. Primordial black holes as a cosmological test of grand unification. Phys. Lett. B 1980, 97, 383-387. [CrossRef]

191. Polnarev, A.G.; Khlopov, M.Y. Cosmology, primordial black Holes, and supermassive particles. Sov. Phys. Uspekhi 1985, 28, 213-232. [CrossRef]

192. Polnarev, A.G.; Khlopov, M.Y. Primordial black holes and the ERA of superheavy particle dominance in the early universe. Sov. Astron. 1981, 25, 406-411.

193. Polnarev, A.G.; Khlopov, M.Y. Dustlike stages in the early universe and constraints on the primordial black-hole spectrum. Sov. Astron. 1982, 26, 391-395.

194. Khlopov, M.Y.; Polnarev, A.G. Superheavy particles in cosmology and evolution of inhomogeneities in the early Universe. In The Very Early Universe; Gibbons, G., Hawking, S., Siclos, E., Eds.; Cambridge University Press: Cambridge, UK, 1983 ; pp. 407-432. 
195. Bullock, J.S.; Primack, J.R. NonGaussian fluctuations and primordial black holes from inflation. Phys. Rev. D 1997, 55, 7423-7439. [CrossRef]

196. Khlopov, M.Y.; Malomed, B.A.; Zel'dovich, Y.B. Gravitational instability of scalar fields and formation of primordial black holes. Mon. Not. R. Astron. Soc. 1985, 215, 575-589. [CrossRef]

197. Zeldovich, Y.B.; Podurets, M.A. The evolution of a system of gravitationally interacting point masses. Sov. Astron. 1965, 9 , 742-748.

198. Gurzadian, V.G.; Savvidi, G.K. Collective relaxation of stellar systems. Astrophys. J. 1986, 160, $203-210$.

199. Kalashnikov, O.K.; Khlopov, M.Y. On the possibility of checking the cosmology of asymptotically free SU(5) theory. Phys. Lett. 1983, 127B, 407-422. [CrossRef]

200. Kadnikov, A.F.; Maslyankin, V.I.; Khlopov, M.Y. Modelling of evolution of quasi-stellar systems, formed by particles and antiparticles in early Universe. Astrophysics 1990, 31, 523-531. [CrossRef]

201. Kofman, L.A.; Linde, A.D. Generation of density perturbations in the inflationary cosmology. Nucl. Phys. B 1987, 282, 555-626. [CrossRef]

202. Sakharov, A.S.; Khlopov, M.Y. Cosmological signatures of family symmetry breaking in multicomponent inflation models. Phys. Atom. Nucl. 1993, 56, 412-417.

203. Berezhiani, Z.; Khlopov, M.Y. Theory of broken gauge symmetry of families. Sov. J. Nucl. Phys. 1990, 51, 739-746.

204. Berezhiani, Z.; Khlopov, M.Y. Physical and astrophysical consequences of breaking of the symmetry of families. Sov. J. Nucl. Phys. 1990, 51, 935-942.

205. Berezhiani, Z.; Khlopov, M.Y.; Khomeriki, R.R. On the possible test of quantum flavor dynamics in the searches for rare decays of heavy particles. Sov. J. Nucl. Phys. 1990, 52, 344-347.

206. Sakharov, A.S.; Khlopov, M.Y. Horizontal unification as the phenomenology of the theory of "everything". Phys. Atom. Nucl. 1994, 57, 651-658.

207. Watkins, R.; Widrow, M. Aspects of reheating in first order inflation. Nucl. Phys. B 1992, 374, 446-468. [CrossRef]

208. Hawking, S.W.; Moss, I.G.; Stewart, J.M. Bubble collisions in the very early universe. Phys. Rev. D 1982, 26, 2681-2693. [CrossRef]

209. Moss, I.G. Singularity formation from colliding bubbles. Phys. Rev. D 1994, 50, 676-681. [CrossRef] [PubMed]

210. Konoplich, R.V.; Rubin, S.G.; Sakharov, A.S.; Khlopov, M.Y. Formation of black holes in first-order phase transitions in the Universe. Astron. Lett. 1998, 24, 413-417.

211. Khlopov, M.Y.; Konoplich, R.V.; Rubin, S.G.; Sakharov, A.S. First-order phase transitions as a source of black holes in the early universe. Gravit. Cosmol. 2000, 6, 153-156.

212. Konoplich, R.V.; Rubin, S.G.; Sakharov, A.S.; Khlopov, M.Y. Formation of black holes in the first order phase transitions as cosmological test of mechanisms of symmetry breaking. Phys. Atom. Nucl. 1999, 62, 1593-1600.

213. La, D.; Steinhardt, P.J. Extended inflationary cosmology. Phys. Rev. Lett. 1989, 62, 376-378. [CrossRef]

214. Holman, R.; Kolb, E.W.; Wang, Y. Gravitational couplings of the inflaton in extended inflation. Phys. Rev. Lett. 1990, 62, 17-20. [CrossRef]

215. Holman, R.; Kolb, E.W.; Vadas, S.L.; Wang, Y. Scale invariant extended inflation. Phys. Rev. D 1991, 43, 3833-3845. [CrossRef] [PubMed]

216. Adams, F.C.; Freese, K. Double field inflation. Phys. Rev. D 1991, 43, 353-361. [CrossRef] [PubMed]

217. Copeland, E.J.; Liddle, A.R.; Lyth, D.H.; Stewart, E.D.; Wands, D. False vacuum inflation with Einstein gravity. Phys. Rev. D 1994, 49, 6410-6433. [CrossRef]

218. Occhionero, F.; Amendola, L. Primordial bubbles from quadratic gravity. Phys. Rev. D 1994, 50, 4846-4852. [CrossRef] [PubMed]

219. Amendola, L.; Baccigalupi, C.; Konoplich, R. Reconstruction of the bubble nucleating potential. Phys. Rev. D 1996, 54, 7199-7206. [CrossRef] [PubMed]

220. Turner, M.S.; Weinberg, E.J.; Widrow, L.M. Bubble nucleation in first order inflation and other cosmological phase transitions. Phys. Rev. D 1992, 46, 2384-2403. [CrossRef] [PubMed]

221. Hawking, S.W. Particle creation by black holes. Comm. Math. Phys. 1975, 43, 199-220. [CrossRef]

222. Hawking, S.W. Black hole explosions. Nature 1974, 248, 30-31. [CrossRef]

223. Carr, B.J.; Gilbert, J.H.; Lidsey, J.E. Black hole relics and inflation: Limits on blue perturbation spectra. Phys. Rev. D 1994, 50, 4853-4867. [CrossRef] [PubMed]

224. Barrow, J.D.; Copeland, E.J.; Liddle, A.R. The cosmology of black hole relics. Phys. Rev. D 1992, 46, 645-657. [CrossRef]

225. Alexeyev, S.O.; Pomazanov, M.V. Black hole solutions with dilatonic hair in higher curvature gravity. Phys. Rev. D 1997, 55, 2110-2118. [CrossRef]

226. Dymnikova, I.G. De sitter-schwarzschild black hole: Its particlelike core and thermodynamical properties. Int. J. Mod. Phys. D 1996, 5, 529-540. [CrossRef]

227. Miyama, S.; Sato, K. The upper bound of the number density of primordial black holes from the big bang nucleosynthesis. Prog. Theor. Phys. 1978, 59, 1012-1013. [CrossRef]

228. Zeldovich, Y.B.; Starobinsky, A.A. Possibility of a cold cosmological singularity in the spectrum of primordial black holes. JETP Lett. 1976, 24, 571-573.

229. Naselsky, P.D. Hydrogen recombination kinetics in the presence of low-mass primordial black holes. Sov. Astron. Lett. 1978, 4 , 209-211. [CrossRef] 
230. Lindley, D. Primordial black holes and the deuterium abundance. Mon. Not. R. Astron. Soc. 1980, 193, 593-601. [CrossRef]

231. Zeldovich, Y.B.; Starobinskii, A.A.; Khlopov, M.I.; Chechetkin, V.M. Primordial black holes and the deuterium problem. Sov. Astron. Lett. 1977, 3, 110-112.

232. Rothman, T.; Matzner, R. Upper limits on micro-mini black holes. Astrophys. Space Sci. 1981, 75, 229-236. [CrossRef]

233. MacGibbon, J.H.; Carr, B.J. Cosmic rays from primordial black holes. Astrophys. J. 1991, 371, 447-469. [CrossRef]

234. Lewicki, M.; Vaskonen, V. On bubble collisions in strongly supercooled phase transitions. Phys. Dark Univ. 2020, $30,100672$. [CrossRef]

235. Dymnikova, I.; Khlopov, M.Y.; Koziel, L.; Rubin, S.G. Quasilumps from first-order phase transitions. Gravit. Cosmol. 2000, 6, 311-318.

236. Chechetkin, V.M.; Khlopov, M.Y.; Sapozhnikov, M.G. Antiproton interactions with light elements as a test of GUT cosmology. Riv. Nuovo Cim. 1982, 5, 1-79. [CrossRef]

237. MacGibbon, J.H.; Carr, B.J. Cosmic rays from primordial black holes and constraints on the early universe. Phys. Rep. 1998, 307, 141-154. [CrossRef]

238. Liddle, A.R.; Green, A.M. Cosmological constraints from primordial black holes. Phys. Rep. 1998, 307, 125-131. [CrossRef]

239. Khlopov, M.Y.; Chechetkin, V.M. Antiprotons in the universe as a cosmological test of grand unification. Sov. J. Part. Nucl. 1987, 18, 267-288.

240. Carr, B.; Kuhnel, F. Primordial Black Holes as Dark Matter: Recent Development. Ann. Rev. Nucl. Part. Sci. 2020, 70, 355-394. [CrossRef]

241. Kroupa, P.; Haslbauer, M.; Banik, I.; Nagesh, S.T.; Pflamm-Altenburg, J. Constraints on the star formation histories of galaxies in the Local Cosmological Volume. Mon. Not. R. Astr. Soc. 2020, 497, 37. [CrossRef]

242. Chae, K.-H.; Lelli, F.; Desmond, H.; McGaugh, S.S.; Li, P.; Schombert, J.M. Testing the Strong Equivalence Principle: Detection of the External Field Effect in Rotationally Supported Galaxies. Astrophys. J. 2020, 904, 51. [CrossRef]

243. Sakharov, A.D. Cosmoparticle physics-A multidisciplinary science. Vestn. SSSR 1989, 4, 39-40. 